\title{
PARAMETRIC DUALITY AND KERNELIZATION: LOWER BOUNDS AND UPPER BOUNDS ON KERNEL SIZE*
}

\author{
JIANER CHEN ${ }^{\dagger}$, HENNING FERNAU ${ }^{\ddagger}$, IYAD A. KANJ§, AND GE XIA
}

\begin{abstract}
Determining whether a parameterized problem is kernelizable and has a small kernel size has recently become one of the most interesting topics of research in the area of parameterized complexity and algorithms. Theoretically, it has been proved that a parameterized problem is kernelizable if and only if it is fixed-parameter tractable. Practically, applying a data reduction algorithm to reduce an instance of a parameterized problem to an equivalent smaller instance (i.e., a kernel) has led to very efficient algorithms and now goes hand-in-hand with the design of practical algorithms for solving $\mathcal{N} \mathcal{P}$-hard problems. Well-known examples of such parameterized problems include the VERTEX COVER problem, which is kernelizable to a kernel of size bounded by $2 k$, and the PLANAR DOMINATING SET problem, which is kernelizable to a kernel of size bounded by $335 k$. In this paper we develop new techniques to derive upper and lower bounds on the kernel size for certain parameterized problems. In terms of our lower bound results, we show, for example, that unless $\mathcal{P}=\mathcal{N} \mathcal{P}$, PLANAR VERTEX COVER does not have a problem kernel of size smaller than $4 k / 3$, and PLANAR INDEPENDENT SET and PLANAR DOMINATING SET do not have kernels of size smaller than $2 k$. In terms of our upper bound results, we further reduce the upper bound on the kernel size for the PLANAR DOMINATING SET problem to $67 k$, improving significantly the $335 k$ previous upper bound given by Alber, Fellows, and Niedermeier [J. ACM, 51 (2004), pp. 363-384]. This latter result is obtained by introducing a new set of reduction and coloring rules, which allows the derivation of nice combinatorial properties in the kernelized graph leading to a tighter bound on the size of the kernel. The paper also shows how this improved upper bound yields a simple and competitive algorithm for the PLANAR DOMINATING SET problem.
\end{abstract}

Key words. parameterized algorithm, planar graph, dominating set, vertex cover, independent set, kernel

AMS subject classifications. 05C85, 68Q17

DOI. $10.1137 / 050646354$

1. Introduction. Many practical algorithms for $\mathcal{N} \mathcal{P}$-hard problems start by applying data reduction subroutines to the input instances of the problem. The hope is that after the data reduction phase the instance of the problem has shrunk to a moderate size. This makes the applicability of a second phase, such as a branchand-bound phase, to the resulting instance more feasible. Weihe showed in [41] how a practical preprocessing algorithm for a variation of the DOMINATING SET problem, called the RED/BLUE DOMINATING SET problem, resulted in breaking up input instances of the problem into much smaller instances. Abu-Khzam, Langston, and Shanbhag [2], in their implementation of algorithms for the VERTEX COVER problem,

* Received by the editors November 30, 2005; accepted for publication (in revised form) April 11, 2007; published electronically November 14, 2007. An extended abstract of this paper appeared in STACS 2005.

http://www.siam.org/journals/sicomp/37-4/64635.html

$\dagger$ Department of Computer Science, Texas A\&M University, College Station, TX 77843 (chen@cs. tamu.edu). The research of this author was supported in part by NSF grants CCF-0430683 and CCR-0311590.

${ }^{\ddagger}$ FB 4-Abteilung Informatik, Universität Trier, D-54286 Trier, Germany (fernau@informatik. uni-trier.de).

$\S$ Corresponding author. School of CTI, DePaul University, 243 S. Wabash Avenue, Chicago, IL 60604 (ikanj@cs.depaul.edu). The work of this author was supported in part by a DePaul University competitive research grant.

【Department of Computer Science, Acopian Engineering Center, Lafayette College, Easton, PA 18042 (gexia@cs.lafayette.edu). 
observed the following: "In many cases, reduction was so effective that it eliminated the core completely, and with it the need for decomposition and search." Similar success was reported with DOMINATING SET as well [3].

On the other hand, many applications seek solutions of very small sizes to fairly large input instances of $\mathcal{N} \mathcal{P}$-hard problems. This has been the main concern for the area of parameterized computation. A parameterized problem is a set of instances of the form $(x, k)$, where $x$ is the input instance and $k$ is a nonnegative integer called the parameter. A parameterized problem is said to be fixed-parameter tractable [17] if there is an algorithm that solves the problem in time $f(k)|x|^{c}$, where $c$ is a fixed constant and $f(k)$ is a recursive function. The development of efficient parameterized algorithms has provided a new approach for practically solving problems that are theoretically intractable. For example, parameterized algorithms for the $\mathcal{N} \mathcal{P}$-hard problem VERTEX COVER [9, 13] have found applications in biochemistry [10], and variants thereof are applicable to problems arising in chip manufacturing [11, 21, 24], while parameterized algorithms in computational logic [35] have provided an effective method for solving practical instances of the ML TYPE-CHECKING problem, which is complete for the class ExpTIME [30].

The notion of a parameterized problem being parameterized tractable, and of the problem having a good data reduction algorithm, turns out to be very closely related. Informally speaking, a kernelization - precisely defined below - is a data reduction procedure that reduces an instance of the problem to another (smaller) instance called the kernel.

It has been proved that a parameterized problem is fixed-parameter tractable if and only if the problem is kernelizable [18].

Designing efficient parameterized algorithms and constructing kernels of reasonable sizes have recently been two of the main topics of research in the area of parameterized computation. More specifically, constructing a problem kernel has become one of the main components in the design of an efficient parameterized algorithm for a problem $[9,11,12,13]$, and designing efficient parameterized algorithms for a parameterized problem now goes hand-in-hand with the construction of a problem kernel of a moderate size for the problem. Two of the most celebrated problems that have been receiving a lot of attention recently from both perspectives are the VERTEX COVER and PLANAR DOMinating SET problems. After a long sequence of algorithms, the VERTEX COVER problem can be solved in time $O\left(1.274^{k}+k n\right)$ [14]. Moreover, the VERTEX COVER problem enjoys a kernel of size bounded by $2 k$, and reducing this bound further seems to be a very challenging task, since it would probably lead to an approximation algorithm for the problem of ratio smaller than 2-a result believed by many people to be unlikely. The PlANAR DOMINATING SET problem as well has undergone some extensive study which culminated in a recent algorithm solving the problem in time $O\left(2^{15.13 \sqrt{k}}+n^{3}\right)$ [25]. Recently, and after many strenuous efforts, it was shown that the PLANAR DOMINATING SET problem has a problem kernel of size $335 k$ that is computable in $O\left(n^{3}\right)$ time [5]. The question of whether such a bound on the problem kernel could be significantly improved remains open.

In this paper we develop new techniques to derive upper and lower bounds on the kernel size for certain parameterized problems. We define the notion of duality of a parameterized problem. Many parameterized tractable problems are the dual of parameterized intractable problems (see $[19,34,38]$ ). As an example, consider the VERTEX COVER and INDEPENDENT SET problems. If $n$ denotes the number of vertices in the whole graph $G$, then it is well known that $(G, k)$ is a YES-instance of vERTEX 
COVER if and only if $\left(G, k_{d}\right)$, where $k_{d}=n-k$, is a YES-instance of INDEPENDENT SET. In this sense, INDEPENDENT SET is the parametric dual problem of VERTEX COVER. While VERTEX COVER is fixed-parameter tractable on general graphs, INDEPENDENT SET is not [17]. Similarly, while DOMINATING SET is fixed-parameter intractable on general graphs, its parametric dual, called NONBLOCKER, is fixed-parameter tractable; see [15]. The landscape changes when we turn our attention towards special graph classes, e.g., problems on planar graphs [6]. Here, for example, both INDEPENDENT SET and DOMINATING SET are fixed-parameter tractable. In fact, and in contrast to what was stated above, there are very many problems for which both the problem itself and its dual are fixed-parameter tractable. This is also true for problems on graphs of bounded genus, as well as on graphs of bounded degree.

The beauty of problems which, together with their dual problems, are fixedparameter tractable is that this constellation allows for, from an algorithmic standpoint, a two-sided attack on the original problem. This two-sided attack enabled us to derive lower bounds on the kernel size for such problems (under classical complexity assumptions). For example, we show that unless $\mathcal{P}=\mathcal{N} \mathcal{P}$, PLANAR VERTEX COVER does not have a kernel of size smaller than $4 k / 3$, and PLANAR INDEPENDENT SET and PLANAR DOMINATING SET do not have kernels of size smaller than $2 k$. To the authors' knowledge, this is the first group of results establishing lower bounds on the kernel size of parameterized problems. We also show that some lower bound results obtained using the techniques devised in this paper are sharp by exhibiting a family of graph classes on which the lower bound on the kernel size of the restricted $\mathcal{N} \mathcal{P}$-hard VERTEX COVER problem approaches the upper bound $2 k$ with an arbitrary precision.

Whereas the lower bounds on the kernel size for PLANAR VERTEX COVER and PLANAR INDEPENDENT SET come close to the known upper bounds of $2 k$ and $4 k$ on the kernel size for the two problems, respectively, the lower bound derived for PLANAR DOMINATING SET is still very far from the $335 k$ upper bound on the problem kernel, which was given by Alber, Fellows, and Niedermeier [5]. To bridge this gap, we investigate the problem of finding a kernel of smaller size for the PLANAR DOMINATING SET problem and derive better upper bounds on the problem kernel for the problem. We improve the reduction rules proposed in [5] and introduce new rules that color the vertices of the graph, enabling us to observe many new combinatorial properties of its vertices. These properties allow us to prove a much stronger bound on the number of vertices in the reduced graph. We show that the PLANAR DOMINATING SET problem has a kernel of size $67 k$ that is computable in $O\left(n^{3}\right)$ time. This is a significant improvement over the results in [5]. Finally, we show how the resulting bound on the kernel size yields a very simple algorithm for the PLANAR DOMINATING SET problem that beats some previous algorithms for the problem, and whose running time even comes close to some of the recently proposed algorithms.

2. Preliminaries. A graph $G$ is said to be planar if $G$ can be embedded on the plane such that no two edges in $G$ cross. It is well known that deciding whether a graph is planar and constructing a planar embedding of the graph in such case can be done in linear time [31]. The number of edges in a planar graph with $n$ vertices for $n \geq 3$ is bounded by $3 n-6[16]$.

A dominating set in a graph $G$ is a set of vertices $D$ such that every vertex in $G$ is either in $D$ or adjacent to at least one vertex in $D$. The size of a dominating set $D$ is the number of vertices in $D$. A minimum dominating set of $G$ is a dominating set with the minimum size. We will denote by $\gamma(G)$ the size of a minimum dominating set in $G$. The Planar DOMinating SET problem, abbreviated PLANaR-DS henceforth, 
is the following: given a planar graph $G$ and a positive integer $k$, either construct a dominating set for $G$ of size at most $k$ or report that no such dominating set exists. It is well known that the PLANAR-DS problem is $\mathcal{N} \mathcal{P}$-complete [27].

A parameterized problem $P$ is a subset of $\Sigma^{*} \times N$, where $\Sigma$ is a fixed alphabet and $N$ is the set of all nonnegative integers. Therefore, each instance of the parameterized problem $P$ is a pair $(I, k)$, where the second component $k$ is called the parameter. The language $L(P)$ is the set of all YES-instances of $P$. We say that the parameterized problem $P$ is fixed-parameter tractable [17] if there is an algorithm that decides whether an input $(I, k)$ is a member of $L(P)$ in time $f(k)|I|^{c}$, where $c$ is a fixed constant and $f(k)$ is a recursive function independent of the input length $|I|$. The class of all fixed-parameter tractable problems is denoted by FPT.

A mapping $s: \Sigma^{*} \times N \rightarrow N$ is called a size function for a parameterized problem $P$ if

- $0 \leq k \leq s(I, k)$,

- $s(I, k) \leq|I|$, and

- $s(I, k)=s\left(I, k^{\prime}\right)$ for all appropriate $k, k^{\prime}$ (independence). Hence, we can also write $s(I)$ for $s(I, k)$.

A problem $P$ together with its size function $s$ is denoted $(P, s)$. The dual problem $P_{d}$ of $P$ is the problem whose corresponding language (i.e., the set of YES-instances) $L\left(P_{d}\right)=\{(I, s(I)-k) \mid(I, k) \in L(P)\}$. The dual of the dual of a problem (with a given size function) is again the original problem. We give some examples below.

$d$-HITTING SET

Given: A hypergraph $G=(V, E)$ with edge degree bounded by $d$, i.e., for all $e \in E$, $|e| \leq d$.

Parameter: $k$.

Question: Is there a hitting set of size at most $k$, i.e.,

$$
\exists C \subseteq V,|C| \leq k, \quad \forall e \in E, C \cap e \neq \emptyset ?
$$

The special case in which $d=2$ corresponds to the VERTEX COVER problem in undirected graphs. Let $L(d$-HS $)$ denote the language of $d$-HitTing SET. Taking as size function $s(G)=|V|$, it is clear that the dual problem obeys $\left(G, k_{d}\right) \in L\left(d-\mathrm{HS}_{d}\right)$ if and only if $G$ has an independent set of cardinality $k_{d}$.

DOMINATING SET

Given: A (simple) graph $G=(V, E)$.

Parameter: $k$.

Question: Is there a dominating set of size at most $k$, i.e.,

$$
\exists D \subseteq V,|D| \leq k, \quad \forall v \in V \backslash D \exists w \in D,(w, v) \in E ?
$$

Taking as size function $s(G)=|V|$, it is clear that the dual problem obeys $\left(G, k_{d}\right) \in L\left(\mathrm{DS}_{d}\right)$ if and only if $G$ has a nonblocker set (i.e., the complement of a dominating set) of cardinality $k_{d}$.

Generally speaking, it is easy to "correctly" define the dual of a problem for the so-called selection problems as formalized in [7]. The concept of duality is less clear, say, for weighted graph problems (with the slight exception of ROMAN DOMINATION; see [22]). Also, different graph parameterizations like treewidth seem to possess no natural dualization.

A kernelization (reduction) for a parameterized problem $P$ with size function $s$ is a polynomial-time computable reduction which maps an instance $(I, k)$ onto $\left(I^{\prime}, k^{\prime}\right)$ such that $(1) s\left(I^{\prime}\right) \leq g(k)$ ( $g$ is a recursive function), (2) $k^{\prime} \leq k$, and $(3)(I, k) \in L(P)$ 
if and only if $\left(I^{\prime}, k^{\prime}\right) \in L(P)$. $I^{\prime}$ is called the problem kernel of $I$. It is known (see [18]) that a parameterized problem is fixed-parameter tractable if and only if it has a kernelization. Of special interest to us in this paper are problems with linear kernels in which $g(k)=\alpha k$ for some constant $\alpha>0$. Such small kernels are known for many important graph problems restricted to planar graphs.

3. Lower bounds on kernel size. Practice in the study of parameterized algorithms has suggested that improved kernelization can lead to improved parameterized algorithms. Many efforts have been made towards obtaining smaller kernels for wellknown $\mathcal{N} \mathcal{P}$-hard parameterized problems (see, for example, $[5,13,18]$ ). A natural question to ask along this line of research is about the limit of polynomial-time kernelization. In this section we develop techniques for deriving lower bounds on the kernel size for certain well-known $\mathcal{N} \mathcal{P}$-hard parameterized problems.

\subsection{General lower bound results.}

TheOREM 3.1. Let $(P, s)$ be an $\mathcal{N} \mathcal{P}$-hard parameterized problem (with size function s). Suppose that $P$ admits an $\alpha k$ kernelization and its dual $P_{d}$ admits an $\alpha_{d} k_{d}$ kernelization, where $\alpha, \alpha_{d} \geq 1$. If $(\alpha-1)\left(\alpha_{d}-1\right)<1$, then $\mathcal{P}=\mathcal{N} \mathcal{P}$.

Proof. Suppose that the assumption of the theorem is true, and let $r(\cdot)$ denote the assumed linear kernelization reduction for $P$. Similarly, let $r_{d}(\cdot)$ be the linear kernelization reduction for $P_{d}$. Consider the following reduction $R$, which on input $(I, k)$ of $P$ performs the following:

if $k \leq \frac{\alpha_{d}}{\alpha+\alpha_{d}} s(I)$ then compute $r(I, k)$;

else compute $r_{d}(I, s(I)-k)$.

Let $\left(I^{\prime}, k^{\prime}\right)$ be the instance computed by the reduction $R$. If $k \leq \frac{\alpha_{d}}{\alpha+\alpha_{d}} s(I)$, then $s\left(I^{\prime}\right) \leq \alpha k \leq \frac{\alpha \alpha_{d}}{\alpha+\alpha_{d}} s(I)$. Otherwise,

$$
\begin{aligned}
s\left(I^{\prime}\right) & \leq \alpha_{d} k_{d} \\
& =\alpha_{d}(s(I)-k) \\
& <\alpha_{d}\left(s(I)-\frac{\alpha_{d}}{\alpha+\alpha_{d}} s(I)\right) \\
& =\frac{\alpha \alpha_{d}}{\alpha+\alpha_{d}} s(I) .
\end{aligned}
$$

Since $(\alpha-1)\left(\alpha_{d}-1\right)<1$, or equivalently $\frac{\alpha \alpha_{d}}{\alpha+\alpha_{d}}<1$, by repeatedly applying $R$ (at most polynomially many times), the problem $P$ can be solved in polynomial time. This completes the proof.

The condition " $\alpha, \alpha_{d} \geq 1$ " in the previous theorem is not crucial in the light of the following lemma.

LEMMA 3.2. Let $(P, s)$ be a parameterized problem such that $P$ admits a kernelization $r(\cdot)$ with $s(r(I, k)) \leq \alpha k$ for some $\alpha<1$. Then $P$ is in $\mathcal{P}$.

Proof. According to our definition of the size function, we have $s\left(I^{\prime}\right) \geq k^{\prime}$ for each instance $\left(I^{\prime}, k^{\prime}\right)$. This is particularly true for the parameter $k^{\prime}$ of the problem kernel instance $I^{\prime}=r(I, k)$. Therefore, $k^{\prime} \leq \alpha k$ for some $\alpha<1$. By repeated kernelization, we arrive at a problem with an arbitrarily small parameter and, hence, of arbitrarily small size. In fact, we need $\mathcal{O}(\log k)$ many such kernelizations, each of them requiring polynomial time. It follows that the given problem can be decided in polynomial time.

Remark. The assumption that $s(I) \geq k$ is crucial here. As a concrete "counterexample," consider the decision tree problem, specified by $n$ objects $X=\left\{x_{1}, \ldots, x_{n}\right\}$, 
$t$ boolean tests $T=\left\{T_{1}, \ldots, T_{t}\right\}$, and a parameter $k$. In this setting, a decision tree is a binary tree $B$ whose leaves are (uniquely) labeled with objects and whose inner nodes are labeled with tests such that on the path from the root to the leaf labeled $x_{i}$, tests are performed that uniquely distinguish $x_{i}$ from all other objects. The overall length of all paths from the root to each leaf is usually considered as the cost function. The question is whether there exists a decision tree with cost bounded by $k$. This problem is known to be $\mathcal{N} \mathcal{P}$-complete (see [32]).

If $n=2^{\ell}$, the decision tree with optimal cost is surely the complete binary tree (possibly not attainable with the given set of tests), since it is optimally balanced. Hence, we have $k>n \log _{2} n$ (otherwise, an algorithm can simply answer NO); this can be seen as a trivial kernelization algorithm. Therefore, $n \in o(k)$. This can be interpreted as giving the (to our knowledge) first natural parameterized problem having a sublinear kernel. On the other hand, this relation also implies that $s(I, k)<k$ is true here, so that the previous lemma does not lead to a contradiction with the known $\mathcal{N} \mathcal{P}$-hardness result.

The problem here is the seemingly innocuous choice of the size function as being $n=|X|$. Observe that any "reasonable" encoding of an instance would rather use $n \log n$ bits, since each element of $X$ would need to get a name. This way, the problem would disappear.

3.2. Concrete lower bound results. From Theorem 3.1 and assuming $\mathcal{P} \neq$ $\mathcal{N} \mathcal{P}$, we immediately obtain Corollaries $3.3-3.8$.

COROLlaRY 3.3. For any $\epsilon>0$, there is no $(4 / 3-\epsilon) k$ kernel for PLANAR VERTEX COVER.

Proof. The four-color theorem implies a $4 k$-kernelization for PLANAR INDEPENDENT SET, which is the dual problem of PLANAR VERTEX COVER.

COROLlaRY 3.4. For any $\epsilon>0$, there is no $(2-\epsilon) k$ kernel for PLANAR INDEPENDENT SET. This result remains true if we restrict the problem to graphs of maximum degree bounded by three, or even to planar graphs of maximum degree bounded by three (both problems are $\mathcal{N} \mathcal{P}$-hard).

Proof. The general VERTEx COVER problem, which is the dual of the INDEPENDENT SET problem, has a $2 k$-kernelization [13] (based on a result of Nemhauser and Trotter). This kernelization is both planarity and bounded-degree preserving.

COROLlary 3.5. For any $\epsilon>0$, there is no $(3 / 2-\epsilon) k$-kernelization for VERTEX COVER restricted to triangle-free planar graphs (this problem is still $\mathcal{N} \mathcal{P}$-hard $[40$, Chapter 7]).

Proof. Based on a theorem by Grötzsch (which can be turned into a polynomialtime coloring algorithm; see [29]), it is known that planar triangle-free graphs are 3 -colorable. This implies a $3 k$ kernel for INDEPENDENT SET restricted to this graph class, which gives the result. Observe that the mentioned $2 k$-kernelization for VERTEX COVER on general graphs preserves planarity and triangle-freeness, which implies that this restriction of the problem has a $2 k$-kernelization.

COROLlary 3.6. For any $\epsilon>0$, there is no $(335 / 334-\epsilon) k_{d}$ kernel for PlanaR NONBLOCKER.

Proof. A $335 k$ kernel for PLANAR-DS was derived in [5].

Corollary 3.7. For any $\epsilon>0$, there is no $(2-\epsilon) k$ kernel for PLANAR-DS. This remains true when further restricting the graph class to planar graphs of maximum degree three (the problem is still $\mathcal{N} \mathcal{P}$-hard).

Proof. In [20], a $2 k_{d}$-kernelization for NONBLOCKER on general graphs which preserves planarity and degree bounds was derived (see also [37, Thm. 13.1.3]). 
Corollary 3.8 (see [15]). For any $\epsilon>0$, there is no $(2-\epsilon) k$ kernel for DOMINATING SET ON CUBIC GRAPHS. This is interesting, since that case is the best match between upper and lower bounds for domination problems.

The above results open a new line of research and prompt us to ask whether we can find examples of problems such that the derived kernel sizes are optimal (unless $\mathcal{P}=\mathcal{N} \mathcal{P}$ ), and whether we can close the gaps between the upper bounds and lower bounds on the kernel size. According to our previous discussion, PLANAR VERTEX COVER on triangle-free graphs is our "best match": we know how to derive a kernel of size $2 k$ and (assuming $\mathcal{P} \neq \mathcal{N} \mathcal{P}$ ) we know that no kernel smaller than $3 k / 2$ exists. On the other hand, the 335k upper bound on the kernel size for PLANAR-DS [5] is very far from the $2 k$ lower bound proved above. In the next sections, we improve this upper bound to $67 k$ in an effort to bridge the huge gap between the upper bound and lower bound on the kernel size for this problem. This allows us to state the following corollary.

Corollary 3.9. Assuming $\mathcal{P} \neq \mathcal{N} \mathcal{P}$, there is no $(67 / 66-\epsilon) k_{d}$ kernel for PLANAR NONBLOCKER for any choice of $\epsilon>0$.

Remark. Since "Euler-type" theorems exist for graphs of bounded genus $g$, it can be shown that there is a constant $c_{g}$ such that each graph of genus $g$ is $c_{g}$-colorable. Therefore, lower bounds on the kernel sizes of VERTEX COVER on graphs of genus $g$ can be derived. For triangle-free graphs of genus $g$, Thomassen has shown that the corresponding constant $c_{g}^{\prime}$ is in $\mathcal{O}\left(g^{1 / 3}(\log g)^{-2 / 3}\right)$ (see $\left.[28,39]\right)$.

Remark. Recently, Fomin and Thilikos [26] were able to extend the linear kernel result for DOMINATING SET to graphs on surfaces of bounded genus. Therefore, our lower bound results extend to these more general graph classes as well.

3.3. Can we improve on the lower bounds? In the following, we reproduce a construction that is essentially due to Paul Seymour. ${ }^{1}$ This construction shows that the lower bound results obtained using the techniques devised in this section can be sharp for certain problems.

Consider the following family $\mathcal{G}_{n}$ of graph classes. A graph $G$ is in $\mathcal{G}_{n}$ if and only if it satisfies the following two conditions.

1. $G=(V, E)$ can be partitioned into $2 n+1$ mutually disjoint independent sets, i.e., $V=I_{1} \cup \cdots \cup I_{2 n+1}, I_{i} \cap I_{j}=\emptyset$ for all $1 \leq i<j \leq 2 n+1$, and the induced graphs $G\left[I_{i}\right]$ contain no edges.

2. The edge set $E$ can be partitioned into $2 n+1$ groups $E_{1}, \ldots, E_{2 n+1}$ such that

$$
E_{i}=E\left(G\left[I_{i} \cup I_{i \bmod (2 n+1)+1}\right]\right) .^{2}
$$

Figure 1 provides an example of a graph in $\mathcal{G}_{n}$.

Since each of these classes is closed under taking induced subgraphs, we can deduce by the Nemhauser-Trotter kernelization [13] the following lemma.

LEMMA 3.10. VERTEX COVER restricted to $\mathcal{G}_{n}$ admits a kernel of size $2 k$ (within $\mathcal{G}_{n}$ ).

Since the graphs in $\mathcal{G}_{n}$ are "nearly bipartite," we have the following result.

LEMMA 3.11. INDEPENDENT SET restricted to $\mathcal{G}_{n}$ admits a kernel of size $(2+$ $1 / n) k_{d}$ (within $\mathcal{G}_{n}$ ).

Proof. Let $G=(V, E) \in \mathcal{G}_{n}$ with an independent set decomposition $V=I_{1} \cup$ $\cdots \cup I_{2 n+1}$ that certifies this membership. To simplify the notation, we assume that

\footnotetext{
${ }^{1}$ Personal communication (2005).

${ }^{2}$ We assume that the graph $G$ is given with a certificate of membership in $\mathcal{G}_{n}$, which is a partitioning of its vertex set into the $2 n+1$ subsets.
} 


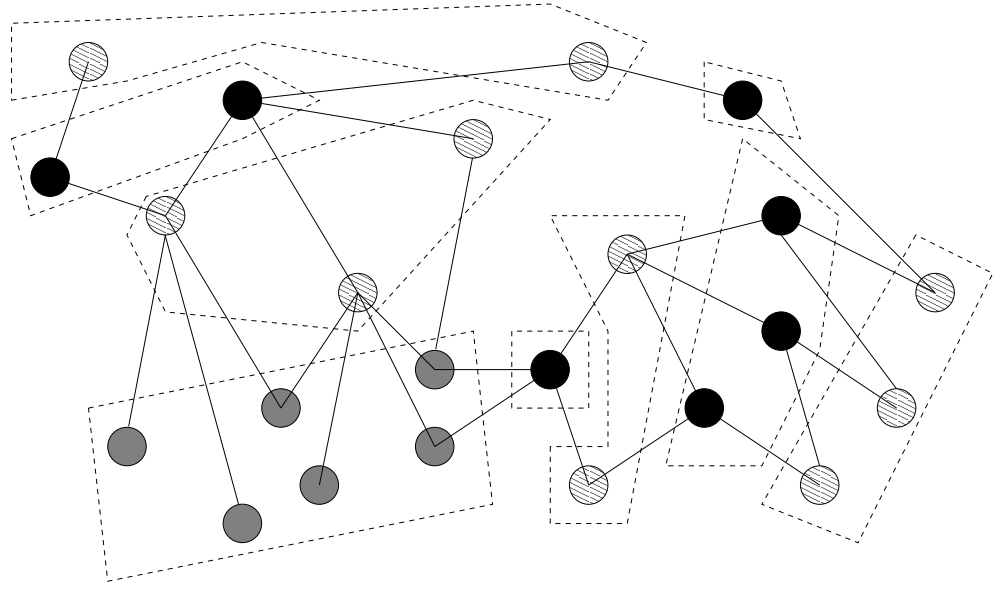

FIG. 1. An example of a graph in $\mathcal{G}_{n}$.

additions and subtractions of indices are all performed modulo $2 n+1$. Consider the sets

$$
J_{i}=I_{i} \cup I_{i+2} \cup \cdots \cup I_{i-3}, \quad 1 \leq i \leq 2 n+1 .
$$

$J_{i}$ greedily collects "every second" set starting at $I_{i}$ so that each set $J_{i}$ forms an independent set of $G$. It can be easily verified that

$$
\sum_{i=1}^{2 n+1}\left|J_{i}\right|=n|V| .
$$

This shows that there exists an index $i$ for which the set $J_{i}$ contains at least a fraction $n /(2 n+1)$ of all the vertices. Moreover, such a $J_{i}$ can be found in polynomial time. Therefore, we can answer YES straightaway whenever we are given a graph $G=$ $(V, E) \in \mathcal{G}_{n}$ with a parameter $k_{d} \leq(n /(2 n+1))|V|$, as an instance of INDEPENDENT SET. This means that we have $|V|<(2+1 / n) k_{d}$ for all the remaining instances.

TheOREM 3.12. For each n, verTEX COVER restricted to $\mathcal{G}_{n}$ is $\mathcal{N} \mathcal{P}$-complete.

Proof. Membership in $\mathcal{N P}$ is inherited from the general verTEx COVER problem. We will show that 3-SAT is polynomial-time reducible to VERTEX COVER restricted to $\mathcal{G}_{n}$. We highlight the main elements in the reduction here and leave the verification of some of the details to the interested reader.

Let $\mathcal{C}=\left\{C_{1}, \ldots, C_{m}\right\}$ be a collection of clauses. Without loss of generality, we can assume that $\left|C_{i}\right|=3$ for all $1 \leq i \leq m$. Let $\ell_{i}^{j}$ refer to the $j$ th literal in clause $C_{i}$, i.e., $\ell_{i}^{j}=y_{i}^{j}$ or $\ell_{i}^{j}=\bar{y}_{i}^{j}$ for some variable $y_{i}^{j} \in X=\left\{x_{1}, \ldots, x_{r}\right\}$.

We construct a graph $G=(V, E) \in \mathcal{G}_{n}$ as follows. For each variable $x_{i}$, we introduce a cycle $\left(v_{i}^{1}, \ldots, v_{i}^{4 n+2}\right)$ of even length. Clearly, $2 n+1$ of these vertices will be in any feasible vertex cover. For each clause $C_{i}$, we introduce a cycle $\left(u_{i}^{1}, \ldots, u_{i}^{2 n+1}\right)$ of odd length. Clearly, $n+1$ of these vertices will be in any feasible vertex cover. Summarizing, the graph described so far will have at least $r(2 n+1)+m(n+1)$ vertices in any feasible cover. Since we will now add more edges to this graph, the lower bound on the size of the vertex cover will still be valid. At the same time, we will maintain the property that $I_{j}=\left\{v_{i}^{j}, v_{i}^{j+2 n+1} \mid 1 \leq i \leq r\right\} \cup\left\{u_{i}^{j} \mid 1 \leq i \leq m\right\}$, where $1 \leq j \leq 2 n+1$, are all independent sets. 
If $\ell_{i}^{1}=y_{i}^{1}=x_{q}$, then we will make $u_{i}^{1}$ adjacent to $v_{q}^{2}$. If $\ell_{i}^{2}=y_{i}^{2}=x_{q}$, then we will make $u_{i}^{2}$ adjacent to $v_{q}^{2 n+2}$. If $\ell_{i}^{3}=y_{i}^{3}=x_{q}$, then we will make $u_{i}^{3}$ adjacent to $v_{q}^{2}$.

If $\ell_{i}^{1}=\bar{y}_{i}^{1}=\bar{x}_{q}$, then we will make $u_{i}^{1}$ adjacent to $v_{q}^{2 n+3}$. If $\ell_{i}^{2}=\bar{y}_{i}^{2}=\bar{x}_{q}$, then we will make $u_{i}^{2}$ adjacent to $v_{q}^{1}$. If $\ell_{i}^{3}=\bar{y}_{i}^{3}=\bar{x}_{q}$, then we will make $u_{i}^{3}$ adjacent to $v_{q}^{2 n+3}$.

Now, if $x_{i}$ is set to true in a satisfying assignment of the given 3-SAT instance, then we put $v_{i}^{j}$ into the vertex cover if and only if $j$ is even. If $x_{i}$ is set to false in a satisfying assignment of the given 3 -SAT instance, then we put $v_{i}^{j}$ into the vertex cover if and only if $j$ is odd. It is not difficult to verify that a satisfying assignment of $\mathcal{C}$ can be translated into a feasible vertex cover of size $r(2 n+1)+m(n+1)$.

The same identification of vertices from $v_{i}^{j}$ with variable settings allows us to translate a feasible vertex cover of size $r(2 n+1)+m(n+1)$ into a satisfying assignment for the given 3-SAT instance.

Corollary 3.13. Unless $\mathcal{P}=\mathcal{N} \mathcal{P}$, the VerTex COVER problem restricted to $\mathcal{G}_{n}$ does not have a kernel of size $(2-\epsilon) k$ for any $\epsilon>0$.

Proof. This follows from Lemma 3.11 and Theorem 3.1.

Remark. The above corollary shows that the lower bound results on the kernel size for VERTEX COVER restricted to $\mathcal{G}_{n}$ obtained using the techniques in this paper are tight. It also shows that it is unlikely that the VERTEX COVER problem on general graphs admits a kernelization of size $(2-\epsilon) k$ with the property that the produced kernel is a subgraph of the original graph, the reason being that such a kernelization would also be a kernelization for the vertex cover problem restricted to the class $\mathcal{G}_{n}$ with the same kernel bound.

3.4. A possible two-sided attack for exact algorithms. With problems having both FPT algorithms for their primal and for their dual parameterizations, we have the possibility of converting both algorithms into a nonparameterized algorithm. This is like attacking the problem from two sides. This means that we can use either of the two FPT algorithms, depending on "to which side" our concrete problem instance is closer.

Theorem 3.14. Let $(P, s)$ be a parameterized problem and $P_{d}$ its dual. Assume that both $P$ and $P_{d}$ are in FPT. Let $f$ be a monotone function. Assume that there is an algorithm $A$ that solves an instance $(I, k)$ of $P$ in time $\mathcal{O}(f(\beta k) p(s(I)))$ for some polynomial $p$, and that $A_{d}$ is an algorithm that solves an instance $\left(I, k_{d}\right)$ of $P_{d}$ in time $\mathcal{O}\left(f\left(\beta_{d} k_{d}\right) p_{d}(s(I))\right)$ for a polynomial $p_{d}$.

Then, there is an algorithm $A^{\prime}$ for solving the nonparameterized problem instance I running in time

$$
\mathcal{O}\left(f\left(\frac{\beta \beta_{d}}{\beta+\beta_{d}} s(I)\right) p^{\prime}(s(I))\right)
$$

for some polynomial $p^{\prime}$.

Proof. The idea is to use algorithm $A$ as long as it is better than using $A_{d}$. This means that we have to compare

$$
f(\beta k) \text { to } f\left(\beta_{d}\left(s(I)-k_{d}\right)\right) .
$$

Since $f$ is monotone, this means we can simply compare

$$
\beta k \text { to } \beta_{d}\left(s(I)-k_{d}\right) .
$$

Some simple algebra shows that we can have the following algorithm $A^{\prime}$ for the nonparameterized problem $P$, given an instance $I$ : 
for all parameter values $k$ do:

if $k \leq \frac{\beta_{d}}{\beta+\beta_{d}} s(I)$ then compute $A(I, k)$;

else compute $A_{d}(I, s(I)-k)$;

output the 'best' of all computed solutions.

Considering the boundary case $k=\frac{\beta_{d}}{\beta+\beta_{d}} s(I)$ gives the claimed worst-case running time. Here, $p^{\prime}(j)=j\left(p(j)+p_{d}(j)\right)$.

Unfortunately, we currently lack good examples that prove this approach superior to published (problem-tailored) exact algorithms.

4. Reduction and coloring rules. In this section we show how to improve the upper bound on the kernel size for the PLANAR-DS problem to $67 k$. In the remainder of the paper we will always assume that the graph we are dealing with is planar.

In this section we present an $O\left(n^{3}\right)$ time preprocessing scheme that reduces the graph $G$ to a graph $G^{\prime}$ such that $\gamma(G)=\gamma\left(G^{\prime}\right)$ and such that given a minimum dominating set for $G^{\prime}$, a minimum dominating set for $G$ can be constructed in linear time. We will color the vertices of the graph $G$ with two colors: black and white. Initially, all vertices are colored black. Informally speaking, white vertices will be those vertices for which we know for sure when we color them that there exists a minimum dominating set for the graph excluding all of them. The black vertices are all other vertices. Note that it is possible for white vertices to be in some minimum dominating set, but the point is that there exists at least one minimum dominating set that excludes all white vertices. Hence, the black-and-white coloring is only an auxiliary structure and not part of the problem definition. We start with the following definitions that are adopted from [5] with minor additions and modifications.

For a vertex $v$ in $G$ denote by $N(v)$ the set of neighbors of $v$, and by $N[v]$ the set $N(v) \cup\{v\}$. By removing a vertex $v$ from $G$, we mean removing $v$ and all the edges incident on $v$ from $G$. For a vertex $v$ in $G$, we partition its set of neighbors $N(v)$ into three sets: $N_{1}(v)=\{u \in N(v) \mid N(u)-N[v] \neq \emptyset\} ; N_{2}(v)=\left\{u \in N(v)-N_{1}(v) \mid\right.$ $\left.N(u) \cap N_{1}(v) \neq \emptyset\right\}$; and $N_{3}(v)=N(v)-\left(N_{1}(v) \cup N_{2}(v)\right)$. For two vertices $v$ and $w$ we define $N(v, w)=N(v) \cup N(w)$ and $N[v, w]=N[v] \cup N[w]$. We partition $N(v, w)$ into three sets: $N_{1}(v, w)=\{u \in N(v, w) \mid N(u)-N[v, w] \neq \emptyset\} ; N_{2}(v, w)=\{u \in N(v, w)-$ $\left.N_{1}(v, w) \mid N(u) \cap N_{1}(v, w) \neq \emptyset\right\}$; and $N_{3}(v, w)=N(v, w)-\left(N_{1}(v, w) \cup N_{2}(v, w)\right)$.

Definition 4.1. Let $G=(V, E)$ be a plane graph. A region $R(v, w)$ between two vertices $v$ and $w$ is a closed subset of the plane with the following properties:

1. The boundary of $R(v, w)$ is formed by two simple paths $P_{1}$ and $P_{2}$ in $G$ which connect $v$ and $w$, and the length of each path is at most three.

2. All vertices that are strictly inside (i.e., not on the boundary) the region $R(v, w)$ are from $N(v, w)$.

For a region $R=R(v, w)$, let $V[R]$ denote the vertices in $R$; i.e.,

$$
V[R]:=\{u \in V \mid u \text { sits inside or on the boundary of } R\} .
$$

Let $V(R)=V[R]-\{v, w\}$.

Definition 4.2. A region $R=R(v, w)$ between two vertices $v$ and $w$ is called simple if all vertices in $V(R)$ are common neighbors of both $v$ and $w$; that is, $V(R) \subseteq$ $N(v) \cap N(w)$.

We introduce the following definition.

Definition 4.3. A region $R=R(v, w)$ between two vertices $v$ and $w$ is called quasi-simple if $V[R]=V\left[R^{\prime}\right] \cup R^{+}$, where $R^{\prime}=R^{\prime}(v, w)$ is a simple region between $v$ and $w$, and $R^{+}$is a set of white vertices satisfying the following conditions. 
1. Every vertex of $R^{+}$sits strictly inside $R^{\prime}$.

2. Every vertex of $R^{+}$is connected to $v$ and not connected to $w$, and is also connected to at least one vertex on the boundary of $R^{\prime}$ other than $v$.

A vertex in $V(R)$ is called a simple vertex if it is connected to both $v$ and $w$; otherwise it is called nonsimple. The set of vertices $R^{+}$, which consists of the nonsimple vertices in $V(R)$, will be referred to as $R^{+}(v, w)$.

For a vertex $u \in V$, denote by $B(u)$ the set of black vertices in $N(u)$ and by $W(u)$ the set of white vertices in $N(u)$. We describe next the reduction and coloring rules to be applied to the graph $G$. The reduction and coloring rules are applied to the graph until the application of any of them does not change the structure of the graph or the color of any vertex in the graph. The first two reduction rules, Rules 1 and 2, are slight modifications of Rules 1 and 2 introduced in [5]. The only difference is that in the current paper they are applied only to black vertices, and not to all the vertices as in [5].

Rule 1 (see [5]). If $N_{3}(v) \neq \emptyset$ for some black vertex $v$, then remove the vertices in $N_{2}(v) \cup N_{3}(v)$ from $G$ and add a new white vertex $v^{\prime}$ and an edge $\left(v, v^{\prime}\right)$ to $G$.

Rule 2 (see [5]). If $N_{3}(v, w) \neq \emptyset$ for two black vertices $v, w$ and if $N_{3}(v, w)$ cannot be dominated by a single vertex in $N_{2}(v, w) \cup N_{3}(v, w)$, then we distinguish the following two cases.

Case 1. If $N_{3}(v, w)$ can be dominated by a single vertex in $\{v, w\}$, then (1) if $N_{3}(v, w) \subseteq N(v)$ and $N_{3}(v, w) \subseteq N(w)$, remove $N_{3}(v, w)$ and $N_{2}(v, w) \cap N(v) \cap N(w)$ from $G$ and add two new white vertices $z, z^{\prime}$ and the edges $(v, z),(w, z),\left(v, z^{\prime}\right),\left(w, z^{\prime}\right)$ to $G$; (2) if $N_{3}(v, w) \subseteq N(v)$ and $N_{3}(v, w) \nsubseteq N(w)$, remove $N_{3}(v, w)$ and $N_{2}(v, w) \cap$ $N(v)$ from $G$ and add a new white vertex $v^{\prime}$ and the edge $\left(v, v^{\prime}\right)$ to $G$; and (3) if $N_{3}(v, w) \subseteq N(w)$ and $N_{3}(v, w) \nsubseteq N(v)$, remove $N_{3}(v, w)$ and $N_{2}(v, w) \cap N(w)$ from $G$ and add a new white vertex $w^{\prime}$ and the edge $\left(w, w^{\prime}\right)$ to $G$.

Case 2. If $N_{3}(v, w)$ cannot be dominated by a single vertex in $\{v, w\}$, then remove $N_{2}(v, w) \cup N_{3}(v, w)$ from $G$ and add two new white vertices $v^{\prime}, w^{\prime}$ and the edges $\left(v, v^{\prime}\right)$, $\left(w, w^{\prime}\right)$ to $G$.

Rule 3. For each black vertex $v$ in $G$, if there exists a black vertex $x \in N_{2}(v) \cup$ $N_{3}(v)$, color $x$ white and remove the edges between $x$ and all other white vertices in $G$.

Rule 4. For every two black vertices $v$ and $w$, if $N_{3}(v, w) \neq \emptyset$, then for every black vertex $x \in N_{2}(v, w) \cup N_{3}(v, w)$ that does not dominate all vertices in $N_{3}(v, w)$, color $x$ white and remove all the edges between $x$ and the other white vertices in $G$.

Rule 5. For every quasi-simple region $R=R(v, w)$ between two vertices $v$ and $w$, if $v$ is black, then for every black vertex $x \in N_{2}(v, w) \cup N_{3}(v, w)$ strictly inside $R$ that does not dominate all vertices in $N_{2}(v, w) \cup N_{3}(v, w)$ strictly inside $R$, color $x$ white and remove all the edges between $x$ and the other white vertices in $G$.

Rule 6. For every two white vertices $u$ and $v$, if $N(u) \subseteq N(v)$ and $u \in N_{2}(w) \cup$ $N_{3}(w)$ for some black vertex $w$, then remove $v$.

Rule 7. For every black vertex $v$, if every vertex $u \in W(v)$ is connected to all the vertices in $B(v)$, then remove all the vertices in $W(v)$ from $G$.

Rule 8. For every two black vertices $v$ and $w$, let $W(v, w)=W(v) \cap W(w)$. If $|W(v, w)| \geq 2$ and there is a degree-2 vertex $u \in W(v, w)$, then remove all vertices in $W(v, w)$ except $u$, add a new degree- 2 white vertex $u^{\prime}$, and connect $u^{\prime}$ to both $v$ and $w$.

Figure 2 illustrates Rules 4, 6, and 8.

A graph $G$ is said to be reduced if every vertex in $G$ is colored white or black and 

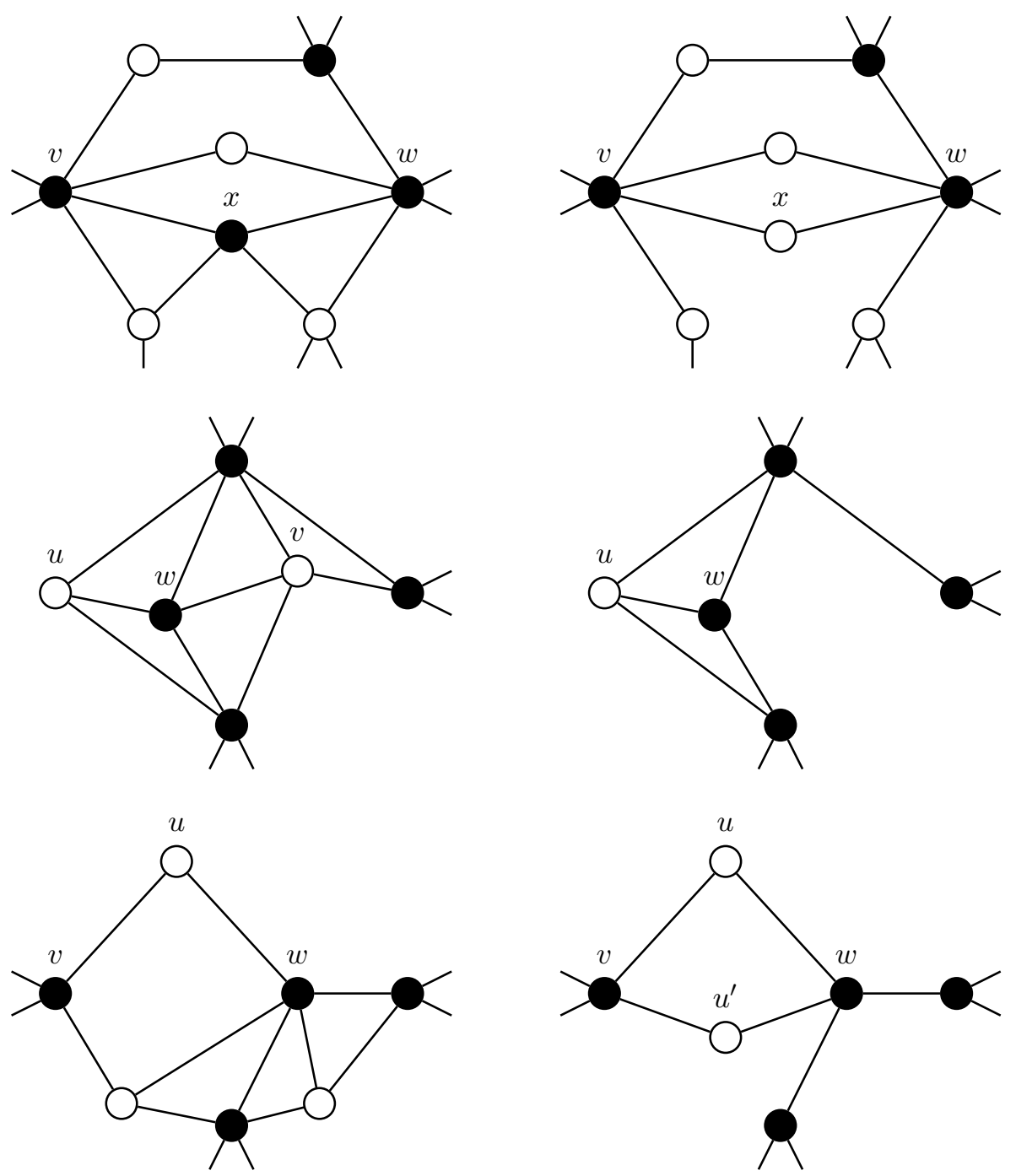

FIG. 2. Illustrations of Rule 4 (top figure), Rule 6 (middle figure), and Rule 8 (bottom figure).

the application of Rules 1-8 leaves the graph $G$ unchanged. That is, the application of any of the above rules does not change the color of any vertex in $G$, nor does it change the structure of $G$. We have the following theorem.

TheOREM 4.4. Let $G$ be a planar graph with $n$ vertices. Then in time $O\left(n^{3}\right)$ we can construct a planar graph $G^{\prime}$ from $G$ such that $(1) G^{\prime}$ is reduced, (2) $\gamma\left(G^{\prime}\right)=$ $\gamma(G),(3)$ there exists a minimum dominating set for $G^{\prime}$ that excludes all white vertices of $G^{\prime}$, and (4) from a minimum dominating set for $G^{\prime}$ a minimum dominating set for $G$ can be constructed in linear time.

Proof. Given a graph $G$, we first color all its vertices black. We then apply Rules 1-8 given above until the application of any of these rules leaves $G$ unchanged. Let $G^{\prime}$ be the resulting graph. Then $G^{\prime}$ is reduced by the definition of a reduced graph. Alber, Fellows, and Niedermeier [5] noted that each successful application of Rules 1 and 2 (i.e., an application that changes the structure of the graph $G$ ) reduces 
the number of vertices in the graph by at least one. Hence, the total number of applications of these two rules is bounded by $n$. By looking at Rules $3-7$, it is easy to see that each of these rules either reduces the number of vertices in $G$ by at least one or changes the color of at least one black vertex to white without adding any new vertices to the graph. Moreover, none of Rules 1-7 increases the number of edges in the graph. If we look at Rule 8, it is not difficult to see that each successful application of this rule reduces the number of edges in the graph by at least 1 . This is true since in a successful application of the rule either $|W(v, w)|>2$ (and in this case the numbers of vertices and edges decrease after the application of the rule) or $|W(v, w)|=2$ and there is a vertex in $W(v, w)$ of degree larger than 2 (otherwise the application of the rule does not change the structure of the graph), and hence the removal of $W(v, w)$ decreases the number of edges in the graph. Noting that the number of edges in a planar graph is linear in the number of vertices and that the application of the rules becomes unnecessary if the graph does not contain any black vertices, we conclude that the total number of successful applications of the operations in Rules 1-8 is $O(n)$. Alber, Fellows, and Niedermeier [5] also showed that Rules 1 and 2 can be executed in time $O\left(n^{2}\right)$ when the graph is planar. Similarly, one can show that Rules 3-8 can also be executed in $O\left(n^{2}\right)$ time (we leave the verification of this simple fact to the interested reader). This, together with the fact that the total number of successful applications of all the rules is $O(n)$, implies that the time needed to construct $G^{\prime}$ is $O\left(n^{3}\right)$.

To show parts (2) and (3) of the theorem, we prove that after the execution of any of the rules, the resulting graph satisfies conditions (2) and (3) in the theorem. The proof will then follow by an inductive argument on the number of applications of the rules. Denote by $H$ the graph before a rule is executed, and by $H^{\prime}$ the resulting graph after the rule is executed. Denote by $D$ a minimum dominating set for $H$ excluding all white vertices in $H$. Initially, $H=G$, and all vertices in $H$ are black. Thus, $H$ trivially satisfies conditions (2) and (3) in the theorem. Suppose now that one of the rules is executed on a graph $H$ satisfying conditions (2) and (3) in the theorem to yield the graph $H^{\prime}$. We need to show that $H^{\prime}$ satisfies conditions (2) and (3) as well.

Suppose that Rule 1 is executed. The same argument used in [5] shows that $\gamma(H)=\gamma\left(H^{\prime}\right) .^{3}$ What is left is showing that $H^{\prime}$ has a minimum dominating set consisting only of black vertices. Let $D$ be a minimum dominating set for $H$ consisting of black vertices. Since $N_{3}(v) \neq \emptyset, D$ must contain a vertex in $N_{2}(v) \cup N_{3}(v) \cup\{v\}$. If $D$ contains a vertex in $N_{2}(v) \cup N_{3}(v)$, then clearly this vertex can be replaced by $v$ which is black. Thus we can assume that $D$ contains $v$ and no vertex in $N_{3}(v) \cup N_{2}(v)$. Then $D$ is also a dominating set for $H^{\prime}$ consisting only of black vertices, and since $\gamma(G)=\gamma(H)=\gamma\left(H^{\prime}\right), D$ is a minimum dominating set for $H^{\prime}$. It follows that $H^{\prime}$ satisfies conditions (2) and (3). The proof of Rule 2 is of the same flavor.

If Rule 3 is executed, then the black vertices in the set $N_{2}(v) \cup N_{3}(v)$, where $v$ is black, will be colored white, and the edges between the white vertices are removed. It suffices to show that after the coloring of one vertex $x$ in $N_{2}(v) \cup N_{3}(v)$ white and removing the edges between $x$ and the other white vertices, conditions (2) and (3) still hold (the same argument can then be applied repetitively to every such vertex). By our inductive hypothesis, before the application of Rule 3 to $H, H$ had a minimum dominating set $D$ of size equal to $\gamma(G)$ that excludes all white vertices in $H$. If $D$ contains $x$, we can replace $x$ by $v$ and have a minimum dominating set of $H$ consisting only of black vertices in $H$. Thus, we can assume, without loss of generality, that $D$

\footnotetext{
${ }^{3}$ The fact that this statement holds true can be easily verified by the reader.
} 
does not include $x$. Since $x$ is the only vertex whose color has changed to white, $D$ consists only of black vertices in $H^{\prime}$. Moreover, it is not difficult to see that $D$ is also a dominating set in $H^{\prime}$ since the edges removed from $H$ are not used to dominate any vertices in $H$ (these edges were incident on vertices that are not in $D$ ). Since by removing edges from the graph the size of the minimum dominating set can only increase, we conclude that $D$ is a minimum dominating set for $H^{\prime}$ excluding all white vertices, and $\gamma\left(H^{\prime}\right)=\gamma(H)=\gamma(G)$.

Suppose Rule 4 is executed. Similarly, we need only show that conditions (2) and (3) still hold after a vertex $x$ has been colored white. If $D$ contains $x$, then by the assumption in Rule $4, x$ does not dominate all the vertices in $N_{3}(v, w)$, and $D$ must also contain at least another vertex $x^{\prime}$ in $N_{2}(v, w) \cup N_{3}(v, w) \cup\{v, w\}$ to dominate $N_{3}(v, w)$. This is true since only vertices in $N_{2}(v, w) \cup N_{3}(v, w) \cup\{v, w\}$ can dominate vertices of $N_{3}(v, w)$. In such a case we can replace $x$ and $x^{\prime}$ by $v$ and $w$ and have a minimum dominating set that consists only of black vertices in $H$. Since $x$ is the only vertex whose color has changed to white, $D$ excludes all white vertices in $H^{\prime}$. It is easy to see that the edges that connect white vertices in $H$ are not used by $D$ to dominate any vertex. By an argument similar to the above, it follows that $D$ is a minimum dominating set for $H^{\prime}$ excluding all white vertices in $H^{\prime}$, and $\gamma\left(H^{\prime}\right)=|D|=\gamma(H)=\gamma(G)$.

Suppose Rule 5 is executed and a vertex $x$ is colored white. Let $R=R(v, w)$ be the quasi-simple region that was being processed in the rule, and note that all the vertices in $R^{+}(v, w)$ are connected to $v$. Let the boundary of $R$ be $(v, y, w, z, v)$. Let $D$ be a dominating set for $H$ consisting only of black vertices. If $D$ contains $x$, then by the assumption in Rule $5, x$ does not dominate all the vertices in $N_{2}(v, w) \cup$ $N_{3}(v, w)$ strictly inside $R$, and $D$ must contain another black vertex $x^{\prime} \in R(v, w)$ in $N_{2}(v, w) \cup N_{3}(v, w) \cup\{v, w, y, z\}$ to dominate the vertices in $N_{2}(v, w) \cup N_{3}(v, w)$ that are strictly inside $R$. Observe that, by the definition of a quasi-simple region, the only vertex that can be dominated by $x$ and not by $v$ is $w$. We distinguish the following cases.

Case 1. $x^{\prime}=v$. Since at least one vertex $r \in\{y, w, z\}$ must be black ( $w$ is connected to both $y$ and $z$, and no edges exist between white vertices; thus it is not possible for all the vertices in $\{y, w, z\}$ to be white) and since all the vertices in $\{y, w, z\}$ dominate $w$, we can replace $x$ by $r$ (note that $x$ is dominated by $v$ ) to obtain a dominating set consisting of black vertices that excludes $x$.

Case 2. $x^{\prime} \neq v$. If $x^{\prime}$ does not dominate $w$, then $x^{\prime}$ must be one of those vertices in $R^{+}$that connect only to $v$ and to the vertices on the boundary of $R$ other than $w$. Since all such boundary vertices are dominated by $v$, and $x^{\prime}$ is dominated by $v$ as well, we can replace $x^{\prime}$ by $v$ in $D$, and the case reduces to Case 1 above. If $x^{\prime}$ dominates $w$, then we can replace $x$ by $v$ to get a dominating set consisting of black vertices that excludes $x$.

Thus, we can assume, without loss of generality, that $D$ does not include $x$. Since $x$ is the only vertex whose color has changed to white, $D$ consists only of black vertices in $H^{\prime}$. By an argument similar to that above, it follows that $D$ is a minimum dominating set for $H^{\prime}$ excluding all white vertices in $H^{\prime}$, and $\gamma\left(H^{\prime}\right)=|D|=\gamma(H)=\gamma(G)$.

Suppose Rule 6 is executed and a vertex $v$ is removed as described in the rule. Let $D$ be a minimum dominating set for $H$ excluding all white vertices in $H$. Thus $D$ does not contain $v$. Since $v$ is the only vertex removed, and no vertices are colored, it follows that $D$ is a dominating set for $H^{\prime}$ excluding all white vertices in $H^{\prime}$. What is left is proving that $D$ is a minimum dominating set for $H^{\prime}$. Suppose that $H^{\prime}$ has a 
minimum dominating set $D^{\prime}$ of size strictly smaller than $D$. Then $D^{\prime}$ has to cover $u$, and hence $D^{\prime}$ contains either $u$ or a neighbor of $u$ in $H^{\prime}$. If $D^{\prime}$ contains $u$, since every neighbor of $u$ is also a neighbor of $w$, and $u$ is a neighbor of $w,\left(D^{\prime} \cup\{w\}\right)-\{u\}$ is a minimum dominating set for $H$ of size smaller than $D$, a contradiction (note that since $w$ is a neighbor of $u, w$ is a neighbor of $v$ as well and hence dominates $v$ ). On the other hand, if $D^{\prime}$ contains a neighbor of $u$, since $N(u) \subseteq N(v), D^{\prime}$ also contains a neighbor of $v$ in $H$ and hence dominates $v$. Thus, $D^{\prime}$ is a dominating set for $H$ of size smaller than $D$, a contradiction. It follows that $|D|=\gamma\left(H^{\prime}\right)=\gamma(H)=\gamma(G)$.

Suppose that Rule 7 is executed on a black vertex $v$ and all vertices in $W(v)$ were removed as described in the rule. Let $D$ be a minimum dominating set for $H$ excluding all white vertices in $H$. Thus, $D$ does not contain any vertex in $W(v)$. Since the vertices in $W(v)$ are the only vertices that were removed, and no vertices in the graph were colored, it follows that $D$ is a dominating set for $H^{\prime}$ excluding all white vertices in $H^{\prime}$. What is left is proving that $D$ is a minimum dominating set for $H^{\prime}$. Suppose that $H^{\prime}$ has a minimum dominating set $D^{\prime}$ of size strictly smaller than $D$. Then $D^{\prime}$ has to cover $v$. Hence $D^{\prime}$ contains either $v$ or a neighbor of $v$ in $B(v)$ because all the vertices in $W(v)$ were removed. In either case, $D^{\prime}$ dominates all the removed vertices in $W(v)$ in $H$, since every vertex in $W(v)$ is adjacent to all vertices in $B(v)$. Therefore $D^{\prime}$ is also a dominating set for $H$ of size smaller than $D$, a contradiction. It follows that $|D|=\gamma\left(H^{\prime}\right)=\gamma(H)=\gamma(G)$.

To prove the statement for Rule 8 , let $D$ be a minimum dominating set for $H$ excluding all white vertices in $H$. Again, $D$ is a dominating set for $H^{\prime}$ excluding all white vertices in $H^{\prime}$. Let $D^{\prime}$ be a minimum dominating set for $H^{\prime}$ and suppose, to get a contradiction, that $\left|D^{\prime}\right|<|D|$. Without loss of generality, we can assume that $D^{\prime}$ contains either $v$ or $w$ (or both); otherwise, to dominate $u$ and $u^{\prime}, D^{\prime}$ has to contain both $u$ and $u^{\prime}$, which can be replaced by $v$ and $w$. Now $D^{\prime}$ is also a dominating set for $H$ of smaller size than $D$, a contradiction. It follows that $D$ is a minimum dominating set for $H^{\prime}$ excluding all white vertices in $H^{\prime}$, and $\gamma\left(H^{\prime}\right)=\gamma(H)=\gamma(G)$.

To prove part (4) of the theorem, note the following: (1) from a minimum dominating set for $G^{\prime}$ one can construct in $O(n)$ time a minimum dominating set for $G^{\prime}$ containing only black vertices (this can be achieved by associating, during the reduction phase, with the vertices colored white the black vertices that can replace them) and (2) a minimum dominating set for $G^{\prime}$ consisting only of black vertices is also a minimum dominating set for $G$. This completes the proof.

5. A problem kernel. Let $G$ be a reduced graph, and let $D$ be a minimum dominating set for $G$ consisting of black vertices such that $|D|=k$. In this section, we will show that the number of vertices $n$ in $G$ is bounded by $67 k$. The following definitions are adopted from [5].

Given any dominating set $D$ in a graph $G$, a $D$-region decomposition of $G$ is a set $\Re$ of regions between pairs of vertices in $D$ such that the following hold.

1. For any region $R=R(v, w)$ in $\Re$, no vertex in $D$ is in $V(R)$. That is, a vertex in $D$ can only be an endpoint of a region in $\Re$.

2. No two distinct regions $R_{1}, R_{2} \in \Re$ intersect. However, they may touch each other by having common boundaries.

Note that all the endpoints of the regions in a $D$-region decomposition are vertices in $D$. For a $D$-region decomposition $\Re$, define $V[\Re]=\bigcup_{R \in \Re} V[R]$. A $D$-region decomposition is maximal if there is no region $R$ such that $\Re^{\prime}=\Re \cup R$ is a $D$-region decomposition with $V[\Re] \subsetneq V\left[\Re^{\prime}\right]$.

For a $D$-region decomposition $\Re$, associate a planar graph $G_{\Re}\left(V_{\Re}, E_{\Re}\right)$ with pos- 
sible multiple edges, where $V_{\Re}=D$, and such that there is an edge between two vertices $v$ and $w$ in $G_{\Re}$ if and only if $R(v, w)$ is a region in $\Re$. A planar graph with multiple edges is called thin if there is a planar embedding of the graph such that for any two edges $e_{1}$ and $e_{2}$ between two distinct vertices $v$ and $w$ in the graph, there must exist two more vertices which sit inside the disjoint areas of the plane enclosed by $e_{1}$ and $e_{2}$.

Alber, Fellows, and Niedermeier [5] showed that the number of edges in a thin graph of $n$ vertices is bounded by $3 n-6$. They also showed that for any plane graph $G$ and a dominating set $D$ of $G$, there exists a maximal $D$-region decomposition for $G$ such that $G_{\Re}$ is thin. Since the maximal $D$-region decomposition in [5] starts with any dominating set $D$ and is not affected by the color a vertex can have, the same results in [5] hold true for our reduced graph $G$ whose vertices are colored black/white, and with a minimum dominating set $D$ consisting of only black vertices. The above discussion is summarized in the following proposition.

Proposition 5.1. Let $G$ be a reduced graph and $D$ a dominating set of $G$ consisting of black vertices. Then there exists a maximal $D$-region decomposition $\Re$ of $G$ such that $G_{\Re}$ is thin.

Corollary 5.2. Let $G$ be a reduced graph with a minimum dominating set $D$ consisting of $k$ black vertices, and let $\Re$ be a maximal $D$-region decomposition of $G$ such that $G_{\Re}$ is thin. Then the number of regions in $\Re$ is bounded by $3 k-6$.

Proof. The number of regions in $\Re$ is the number of edges in $G_{\Re}$. Since $G_{\Re}$ has $|D|=k$ vertices, by [5], the number of edges in $G_{\Re}$ is bounded by $3 k-6$.

In the remainder of this section, $\Re$ will denote a maximal $D$-region decomposition of $G$ such that $G_{\Re}$ is thin. Let $u$ and $v$ be two vertices in $G$. We say that $u$ and $v$ are boundary-adjacent if $(u, v)$ is an edge on the boundary of some region $R \in \Re$. For a vertex $v \in G$, denote by $N^{*}(v)$ the set of vertices that are boundary-adjacent to $v$. Note that for a vertex $v \in D$, since $v$ is black, by Rule 3, all vertices in $N_{2}(v) \cup N_{3}(v)$ must be white. Note also that, by the definition of a $D$-region decomposition, all the endpoints of the regions in $\Re$ are vertices in $D$, and hence are colored black.

Proposition 5.3. Let $v \in D$. The following are true.

(a) (Lemma 6, [5].) Every vertex $u \in N_{1}(v)$ is in $V[\Re]$.

(b) The vertex $v$ is an endpoint of a region $R \in \Re$. That is, there exists a region $R=R(x, y) \in \Re$ such that $v=x$ or $v=y$.

(c) Every vertex $u \in N_{2}(v)$ which is not in $V[\Re]$ is connected only to $v$ and to vertices in $N^{*}(v)$.

Proof. The proof of part (a) appears in [5].

To prove (b), suppose, to get a contradiction, that $v$ is not the endpoint of any region in $\Re$. Since $v \in D$, and by the definition of a region, $v$ must be outside every region in $\Re$. Now $v$ must have a vertex in $N_{1}(v)$; otherwise, all vertices in $N(v)$ would be white and hence removed by Rule 7 (we assume, without loss of generality, that $G$ does not contain any isolated vertices). Let $u \in N_{1}(v)$. By part (a) above, $u$ must belong to some region $R=R(x, y)$. Observe that $u$ must be on the boundary of $R$; otherwise $v$ would be a vertex in $V[R]$. Again, by the definition of a region, $u$ is either boundary-adjacent to $x$ or to $y$. Suppose, without loss of generality, that $u$ is boundary-adjacent to $x$. But then the degenerated region formed by $(x, u, v)$ does not cross $\Re$ (it only touches $R$ ), contradicting the maximality of $\Re$.

To prove part (c), let $u$ be a vertex in $N_{2}(v)$ and note that $u$ is white, and suppose that $u$ is connected to a vertex $w \neq v$ such that $w \notin N^{*}(v)$. Note that $w$ must be in $N_{1}(v)$ (otherwise $w$ would be white and $u$ and $w$ cannot be adjacent) and hence, by 
part (a) above, must belong to some region $R=R(x, y)$. Since $u \notin V[\Re], w$ cannot be inside $R$ and hence is on the boundary of $R$. Moreover, by the definition of a region, $w$ must be boundary-adjacent to either $x$ or $y$. Without loss of generality, assume $w$ is boundary-adjacent to $x$. Now $w \notin N^{*}(v)$, so $w$ cannot be boundary-adjacent to $v$, and $x \neq v$. Consider the degenerated region formed by $(v, u, w, x)$. This region cannot cross any region in $\Re$; otherwise it crosses it via $(u, w)$, and $u$ would be in $V[\Re]$. But this contradicts the maximality of $\Re$ since $u \notin V[\Re]$.

Let $x$ be a vertex in $G$ such that $x \notin V[\Re]$. Then by part (b) in Proposition 5.3, $x \notin D$. Thus, $x \in N(v)$ for some black vertex $v \in D \subseteq V[\Re]$. By part (a) in Proposition 5.3, $x \notin N_{1}(v)$, and hence, $x \in N_{2}(v) \cup N_{3}(v)$. By Rule 3, the color of $x$ must be white. Let $R=R(v, w)$ be a region in $V[\Re]$ of which $v$ is an endpoint (such a region must exist by part (b) of Proposition 5.3). We distinguish two cases.

Case A. $x \in N_{3}(v)$. Since $v$ is black, by Rule 1 , this is only possible if $\operatorname{deg}(x)=1$ and $N_{2}(v)=\emptyset$ (in this case $x$ will be the white vertex added by the rule). In such a case it can be easily seen that we can flip $x$ and place it inside $R$ without affecting the planarity of the graph.

Case B. $x \in N_{2}(v)$. Note that in this case $N_{3}(v)=\emptyset$ by Rule 1 (otherwise $N_{2}(v) \cup N_{3}(v)$ would be removed), and $x$ is only connected to $v$ and $N^{*}(v)$ by part (c) of Proposition 5.3. If $\operatorname{deg}(x)=2$, by a similar argument to Case A above, $x$ can be flipped and placed inside $R$.

According to the above discussion, it follows that the vertices in $G$ can be classified into two categories: (1) those vertices that are in $V[\Re]$ and (2) those that are not in $V[\Re]$, which are those vertices of degree larger than 2 that belong to $N_{2}(v)$ for some vertex $v \in D$ and in this case must be connected only to vertices in $N^{*}(v)$. To bound the number of vertices in $G$ we need to bound the number of vertices in the two categories. We start with the vertices in category (2).

Let $O$ denote the set of vertices in category (2). Note that all vertices in $O$ are white, and no two vertices $u$ and $v$ in $O$ are such that $N(u) \subseteq N(v)$. To see why the latter statement is true, note that every vertex in $O$ must be in $N_{2}(w)$ for some black vertex $w \in D$. So if $N(u) \subseteq N(v)$, then by Rule $6, v$ would have been removed from the graph. To bound the number of vertices in $O$, we will bound the number of vertices in $O$ that are in $N_{2}(v)$ where $v \in D$. Let us denote this set by $N^{\dagger}(v)$. Let $N_{\dagger}^{*}(v)$ be the set of vertices in $N^{*}(v)$ that are neighbors of vertices in $N^{\dagger}(v)$. Note that every vertex in $N^{\dagger}(v)$ has degree $\geq 3$ and is connected only to $v$ and to $N_{\dagger}^{*}(v)$, and that no two vertices $x$ and $y$ in $N^{\dagger}(v)$ are such that $N(x) \subseteq N(y)$.

Assumption 5.4. For the sake of counting the number of vertices in $N^{\dagger}(v)$, it is safe to assume that (1) every vertex in $N^{\dagger}(v)$ has degree exactly $3 ;(2)$ no two vertices $x$ and $y$ in $N^{\dagger}(v)$ are such that $N(x) \subseteq N(y)$; and (3) vertices in $N^{\dagger}(v)$ are only connected to $v$ and to vertices in $N_{\dagger}^{*}(v)$.

Proof. Since properties (2) and (3) are already satisfied by the vertices in $N^{\dagger}(v)$, we need only show how we can make the vertices in $N^{\dagger}(v)$ satisfy property (1) without reducing their number and without affecting properties (2) and (3). To satisfy property (1), we will remove some edges between vertices in $N^{\dagger}(v)$ and $N_{\dagger}^{*}(v)$ without affecting the other properties. This can be done as follows. List the vertices in $N^{\dagger}(v)$ in an arbitrary order $\left\langle u_{1}, \ldots, u_{r}\right\rangle$. Start by picking $u_{1}$; then choose any two neighbors of $u_{1}$ in $N_{\dagger}^{*}(v)$ and remove all edges that join $u_{1}$ to all its neighbors other than $v$ and these two chosen neighbors. Inductively, suppose we have processed vertex $u_{i-1}$; we process vertex $u_{i}$ as follows. Pick two neighbors $w_{1}^{i}$ and $w_{2}^{i}$ of $u_{i}$ in $N_{\dagger}^{*}(v)$ such that no vertex in $\left\{u_{1}, \ldots, u_{i-1}\right\}$ has both $w_{1}^{i}$ and $w_{2}^{i}$ as its picked 
neighbors. Delete all the edges that join $u_{i}$ to all vertices other than $v, w_{1}^{i}$, and $w_{2}^{i}$. We need to show that it is always possible to carry out this step. Suppose not, and let $i$ be the smallest index such that this is not possible. It is easy to verify using the facts that every vertex in $N^{\dagger}(v)$ has degree larger than 2, no two vertices $x$ and $y$ are such that $N(x) \subseteq N(y)$, and that $i>3$. Note that it must be the case that $\operatorname{deg}\left(u_{i}\right)>3$; otherwise, since this step cannot be carried out, the only two neighbors of $u_{i}$ other than $v$ must be neighbors of some other vertex $u_{j} \in\left\{u_{1}, \ldots, u_{i-1}\right\}$, and hence we would have $N\left(u_{i}\right) \subseteq N\left(u_{j}\right)$ for some $u_{j} \in\left\{u_{1}, \ldots, u_{i-1}\right\}$, contradicting the properties satisfied by category-(2) vertices as discussed above. Let $a, b, c$ be three neighbors of $u_{i}$ other than $v$. Since this step cannot be carried out successfully, there must exist three distinct vertices $u_{p}, u_{q}, u_{s} \in\left\{u_{1}, \ldots, u_{i-1}\right\}$ such that $\{a, b\} \subset N\left(u_{p}\right)$, $\{a, c\} \subset N\left(u_{q}\right)$, and $\{b, c\} \subset N\left(u_{s}\right)$. Consider the subgraph $H$ of $G$ induced by the set of vertices $\left\{v, u_{p}, u_{q}, u_{s}, u_{i}, a, b, c\right\}$. Then the following is true about the vertices in $H$ : (1) $u_{i}, u_{p}, u_{q}, u_{s}, a, b, c$ are neighbors of $v$ in $H$; (2) $v, a, b, c$ are neighbors of $u_{i}$ in $H$; (3) $v, a, b$ are neighbors of $u_{p}$ in $H$; (4) $v, a, c$ are neighbors of $u_{q}$ in $H$; (5) $v, b, c$ are neighbors of $u_{s}$ in $H$; (6) $v, u_{i}, u_{p}, u_{q}$ are neighbors of $a$ in $H$; (7) $v, u_{i}, u_{p}, u_{s}$ are neighbors of $b$ in $H$; and (8) $v, u_{i}, u_{q}, u_{s}$ are neighbors of $c$ in $H$. Using all this information, it is not difficult to verify that $H$ is nonplanar (identify vertex $a$ with vertex $b$ along the path $\left(a, u_{p}, b\right)$ to obtain a copy of $\left.K_{3,3}\right)$, contradicting the planarity of $G$. This completes the proof.

Proposition 5.5. $\left|N^{\dagger}(v)\right| \leq 3 / 2\left|N_{\dagger}^{*}(v)\right|$.

Proof. To simplify the counting, by Assumption 5.4, we can assume that every vertex in $N^{\dagger}(v)$ has degree exactly 3; no two vertices $x$ and $y$ in $N^{\dagger}(v)$ are such that $N(x) \subseteq N(y)$; and vertices in $N^{\dagger}(v)$ are connected only to $v$ and $N_{\dagger}^{*}(v)$. Let $x$ be the number of vertices in $N_{\dagger}^{*}(v)$, and let $f(x)=\left|N^{\dagger}(v)\right|$. We will show that $f(x) \leq 3 / 2(x-1)$. We proceed by induction on $x$. If $x=1$, it is clear that $f(x)=0 \leq$ $3 / 2(x-1)$ since by Assumption 5.4, each vertex in $N^{\dagger}(v)$ has degree exactly 3 . If $x=2$, then clearly $f(x) \leq 1 \leq 3 / 2(x-1)$ since at most one vertex can be connected to $v$ and the two vertices in $N_{+}^{*}(v)$ without violating properties (1)-(3) in Assumption 5.4. If $x=3$, then $f(x) \leq 3$ since at most three vertices can be connected to $N_{\dagger}^{*}(v)$ without violating properties (1)-(3) in Assumption 5.4, each connected to $v$ and to two other vertices in $N_{\dagger}^{*}(v)$. Inductively, suppose that if $N_{\dagger}^{*}(v)$ contains $y$ vertices with $3 \leq y<x$, then the number of vertices $f(y)$ in $N^{\dagger}(v)$ satisfies $f(y) \leq 3 / 2(y-1)$. Suppose now that $\left|N_{+}^{*}(v)\right|=x$. Let $u$ be a vertex in $N^{\dagger}(v)$, and let $a, b$ be its neighbors in $N_{+}^{*}(v)$. The vertex $u$ is called hollow if the interior of the region enclosed by $(u, a, v, b, u)$ contains no vertices of $N_{\dagger}^{*}(v)$. If every vertex in $N^{\dagger}(v)$ is hollow, then it is clear that $f(x) \leq x \leq 3 / 2(x-1)$ for $x>3$, and the bound $f(x)=x$ is attained when there are $x$ vertices in $N^{\dagger}(v)$, and every vertex $u$ in $N^{\dagger}(v)$ is adjacent to $v$ and the two neighbors $a$ and $b$ in $N_{\dagger}^{*}(v)$ immediately to the left and right in the clockwise (or counterclockwise) ordering, respectively, of $u$ in the embedding. Suppose now that there is a vertex $u \in N^{\dagger}(v)$ such that $u$ is not hollow. The edges $(u, a),(u, v),(u, b)$, $(v, a),(v, b)$ separate the plane into three faces: $F_{1}$ enclosed by the cycle $(u, a, v, u)$, $F_{2}$ enclosed by the cycle $(u, v, b, u)$, and $F_{3}$, the outer face determined by the cycle $(u, a, v, b, u)$. Let $x_{1}$ be the number of vertices in $N_{\dagger}^{*}(v)$ that are in $F_{1}$ including the boundary, $x_{2}$ the number in $F_{2}$, and $x_{3}$ the number in $F_{3}$. Note that $1 \leq x_{1}<x$ since $a \in F_{1}$ and $b \notin F_{1}, 1 \leq x_{2}<x$ since $b \in F_{2}$ and $a \notin F_{2}$, and $2 \leq x_{3}<x$ since $a$ and $b$ are in $F_{3}$ and at least one vertex in $N_{\dagger}^{*}(v)$ is not in $F_{3}$ since $u$ is hollow, and hence the interior of the face $(u, a, v, b, u)$ contains at least one vertex in $N_{\dagger}^{*}(v)$. Moreover, $x_{1}+x_{2}+x_{3}=x+2$, since $a$ and $b$ are the only vertices counted twice 
when we add the vertices in $N_{\dagger}^{*}(v)$ that are in $F_{1}, F_{2}$, and $F_{3}$. Now every vertex in $N^{\dagger}(v)$ is either (1) connected to two vertices in $N_{\dagger}^{*}(v)$ in $F_{1}$, (2) connected to two vertices in $N_{+}^{*}(v)$ in $F_{2}$, or (3) connected to two vertices in $N_{+}^{*}(v)$ in $F_{3}$. Note that vertex $u$ satisfies property (3). Since $x_{1}, x_{2}, x_{3}<x$, by the inductive hypothesis, the number of vertices satisfying (1) is bounded by $f\left(x_{1}\right) \leq 3 / 2\left(x_{1}-1\right)$, the number of vertices satisfying (2) is bounded by $f\left(x_{2}\right) \leq 3 / 2\left(x_{2}-1\right)$, and the number of vertices satisfying (3) is bounded by $f\left(x_{3}\right) \leq 3 / 2\left(x_{3}-1\right)$. Now $f(x) \leq f\left(x_{1}\right)+f\left(x_{2}\right)+f\left(x_{3}\right) \leq$ $3 / 2\left(x_{1}+x_{2}+x_{3}\right)-9 / 2=3 x / 2-3 / 2=3 / 2(x-1)$. This completes the proof.

LEMma 5.6. The number of vertices in category (2) (i.e., the number of vertices not in $V[\Re]$ ) is bounded by $18 k$.

Proof. Let $v$ and $w$ be any two distinct vertices in $D$ and observe the following. First, $N^{\dagger}(v) \cap N^{\dagger}(w)=\emptyset$, because if $u \in N^{\dagger}(v) \cap N^{\dagger}(w)$, then $(v, u, w)$ would be a degenerated region with $u \notin V[\Re]$ contradicting the maximality of $\Re$. Second, from the first observation it follows that $w \notin N_{\dagger}^{*}(v)$ and $v \notin N_{+}^{*}(w)$, and in general no vertex $a \in D$ belongs to $N_{\dagger}^{*}(b)$ for any vertex $b \in D$; otherwise, there exists a vertex $u \in N^{\dagger}(v)$ that is connected to $w$, and hence $u \in N^{\dagger}(v) \cap N^{\dagger}(w)$, contradicting the first observation. Third, $N_{\dagger}^{*}(v) \cap N_{\dagger}^{*}(w)=\emptyset$; otherwise, there exists a vertex $u \in$ $N_{\dagger}^{*}(v) \cap N_{\dagger}^{*}(w)$ that is connected to a category-(2) vertex $a \in N^{\dagger}(v)$ (or $b \in N^{\dagger}(w)$ ), and the degenerated region $(v, a, u, w)$ (or $(w, b, u, v))$ would contain the vertex $a \notin \Re$ (or $b \notin \Re$ ), contradicting the maximality of $\Re$.

Let $B$ be the number of vertices not in $D$ that are boundary-adjacent to vertices in $D$ (i.e., in $N^{*}(v)-D$ for some $v \in D$ ). Combining the above observations with Proposition 5.5, it follows that the number of category-(2) vertices is

$$
\sum_{v \in D}\left|N^{\dagger}(v)\right| \leq \frac{3}{2} \sum_{v \in D}\left|N_{\dagger}^{*}(v)\right| \leq 3 B / 2
$$

According to the definition of a region, each region in $\Re$ has at most six vertices on its boundary, two of which are vertices in $D$. Thus, each region in $\Re$ can contribute with at most four vertices to $B$. Note that from the above discussion no vertex $a \in D$ belongs to $N_{\dagger}^{*}(b)$ for any vertex $b \in D$, and hence the endpoints of the regions do not contribute to $B$. By Corollary 5.2, the number of regions in $\Re$ is bounded by $3 k-6$. It follows that $B \leq 12 k-24$, and hence the number of category-(2) vertices is bounded by $18 k-36<18 k$. This completes the proof.

To bound the number of vertices in category (1), fix a region $R(v, w)$ between $v, w \in D$. We have the following lemma whose proof is technical and based on caseby-case structural analysis. The proof of the lemma appears in the appendix.

Lemma 5.7 (see Lemma A.5 in the appendix). Let $R=R(v, w)$ be a region in $V[\Re]$. The number of vertices in $V(R)$ is bounded by 16 .

TheOREM 5.8. The number of vertices in $G$ is bounded by $67 k$.

Proof. By Lemma 5.6, the number of category-(2) vertices in $G$ is bounded by $18 k$. Using this bound, we can assume that each region in $\Re$ is nice. By Corollary 5.2 , the number of regions in $\Re$ is bounded by $3 k-6$. According to Lemma 5.7, the number of vertices in $V(R)$, where $R \in \Re$ is a nice region, is bounded by 16 . It follows that the number of vertices in $V(\Re)$ is bounded by $48 k-96$. Thus, the number of vertices in $V[\Re]$, and hence in category (1), is bounded by $48 k-96$ plus the number of vertices in $D$ which are the endpoints of the regions in $\Re$. Therefore the number of vertices in $V[\Re]$ is bounded by $49 k-96$, and the total number of vertices in $G$ is bounded by $67 k-96<67 k$. This completes the proof. 
TheOREm 5.9. Let $G$ be a planar graph with $n$ vertices. Then in time $O\left(n^{3}\right)$, computing a dominating set for $G$ of size bounded by $k$ can be reduced to computing a dominating set of size bounded by $k$ for a planar graph $G^{\prime}$ of $n^{\prime}<n$ vertices, where $n^{\prime} \leq 67 k$.

Proof. According to Theorem 4.4, in time $O\left(n^{3}\right)$ we can construct a reduced graph $G^{\prime}$ from $G$, where $\gamma\left(G^{\prime}\right)=\gamma(G)$, and such that a dominating set for $G$ can be constructed from a dominating set for $G^{\prime}$ in linear time. Moreover, the graph $G^{\prime}$ has no more than $n$ vertices. If $G$ has a dominating set of size bounded by $k$, then $G^{\prime}$ has a dominating set of size bounded by $k$ (since $\gamma(G)=\gamma\left(G^{\prime}\right)$ ), and by Theorem 5.9 , we must have $n^{\prime} \leq 67 k$. If this is the case, then we can work on computing a dominating set for $G^{\prime}$ of size bounded by $k$, from which a dominating set for $G$ can be easily computed. If this is not the case, then $G$ does not have a dominating set of size bounded by $k$, and the answer to the input instance is negative. This completes the proof.

6. A simple algorithm. In this section we present a simple algorithm for determining whether a graph $G$ has a dominating set of size bounded by $k$.

Let $G=(V, E)$ be a planar graph given with an embedding in the plane. The layer decomposition of $G$ with respect to the embedding is a partitioning of $V$ into disjoint layers $\left(L_{1}, \ldots, L_{r}\right)$ defined inductively as follows. Layer $L_{1}$ is the set of vertices that lie on the outer face of $G$, and layer $L_{i}$ is the set of vertices that lie on the outer face of $G-\bigcup_{j=1}^{i-1} L_{j}$ for $1<i \leq r$. It is well known that a layer decomposition of a planar graph $G$ can be computed in linear time in the number of vertices in the graph [4].

A separator in a graph $G$ is a set of vertices $S$ whose removal disconnects $G$. If $\left(L_{1}, \ldots, L_{r}\right)$ is a layer decomposition of $G$, then clearly the vertices in any layer $L_{i}$ form a separator in $G$, separating the vertices in layers $L_{1}, \ldots, L_{i-1}$ from those in layers $L_{i+1}, \ldots, L_{r}$. Let $(G, k)$ be an instance of the PLANAR-DS problem. By Theorem 5.9, we can assume that $G$ is reduced and that the number of vertices $n$ of $G$ satisfies $n \leq 67 k$. Let $\left(L_{1}, \ldots, L_{r}\right)$ be a layer decomposition of $G$. Let $c>0$ be a constant which will be determined later, and set $l=\lceil c \sqrt{k}\rceil$. Consider the families of layers $F_{i}, i=1, \ldots, l$, where $F_{i}$ consists of layers $L_{i}, L_{i+l}, L_{i+2 l}, \ldots$ Assume for now that the number of layers $r \geq l$. We will show later how to handle the situation when this is not the case. The families $F_{i}, i=1, \ldots, l$, are disjoint, and each family forms a separator separating the graph into connected components that will be called chunks, where each chunk consists of at most $l$ consecutive layers. Since these $l$ families are disjoint and partition the layers into $l$ groups, and since the graph has at most $67 k$ vertices, there exists an index $1 \leq \mu \leq l$, such that the number of vertices in $F_{\mu}$ is bounded by $67 k / l$. Again, observe that the removal of $F_{\mu}$ from $G$ separates $G$ into chunks, each consisting of at most $l$ consecutive layers. Let these chunks be $G_{1}, \ldots, G_{s}$.

The basic idea behind the algorithm is to apply a simple divide-and-conquer strategy by removing the vertices in the family $F_{\mu}$ to split the graph into chunks, then to compute a minimum dominating set for the resulting chunks using the algorithm introduced in [33], which is a variation of Baker's algorithm [8]. To do this, for each vertex $v$ in the $F_{\mu}$, we "guess" whether $v$ is in the minimum dominating set for $G$ or not (basically, what we mean by guessing is enumerating all sequences corresponding to the different possibilities). For each guess of all the vertices in $F_{\mu}$, we will solve the corresponding instance with respect to that guess. It was shown in [33] how this guessing process can be achieved using at most three statuses per vertex. Hence, guessing the vertices in $F_{\mu}$ can be done by enumerating at most $3^{\left|F_{\mu}\right|} \leq 3^{67 k / l}$ 
ternary sequences. After guessing each vertex in the separator and updating the graph accordingly, the instance becomes an instance of a variation of the minimum dominating set problem due to the constraints placed on some of the vertices in the graph. Kanj and Perković introduced an algorithm in [33], which is a variation of Baker's algorithm [8], to solve this problem. The algorithm introduced in [33] solves this problem on the chunks in time $O\left(27^{d+1} n\right)$, where $d$ is the maximum number of layers in a chunk (i.e., the maximum depth of a chunk). Noting that $d \leq l$ and that $n \leq 67 k$, we conclude that after guessing all the vertices in $F_{\mu}$, the problem can be solved in time $O\left(27^{l} k\right)$. If the number of layers $r$ in $G$ is less than $l$, we can simply call the algorithm in [33] directly on $G$ to solve the problem in time $O\left(27^{l} k\right)$. The algorithm is given in Figure 3 below.

It is not difficult to verify that the running time of the algorithm is $O\left(3^{67 k / l} \cdot 27^{l}\right.$. $k+n^{3}$ ), where the $O\left(n^{3}\right)$ time is the time taken to reduce $G$ to its kernel. Niedermeier and Rossmanith showed how to get rid of the $k$ factor corresponding to the kernel size in the running time of such algorithms [36]. Using their techniques, the running time of the algorithm becomes $O\left(n^{3}+3^{67 k / l} \cdot 27^{l}\right)$. We choose $c$, and hence $l$, so that the above expression is minimized. It can be shown that the expression is minimized when $c=\sqrt{67 / 3}$, and the running time of the algorithm becomes $O\left(n^{3}+2^{45 \sqrt{k}}\right)$.

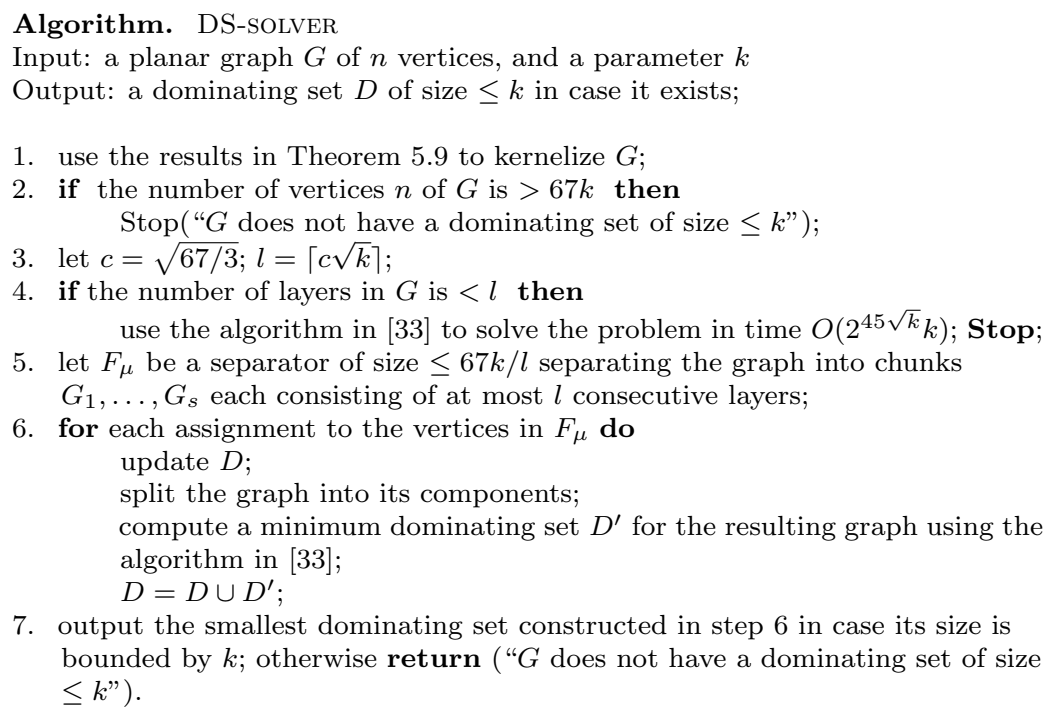

FIG. 3. A simple algorithm solving PLANAR-DS.

TheOREM 6.1. In time $O\left(n^{3}+2^{45 \sqrt{k}}\right)$, it can be determined whether or not a planar graph on $n$ vertices has a dominating set of size bounded by $k$.

Theorem 6.1 shows that our algorithm for solving the PLANAR-DS problem is competitive with the previous algorithms using the similar technique of layer decomposition of a planar graph $[4,33]$. The above algorithm improves the original $O\left(2^{70 \sqrt{k}} n\right)$ time algorithm given in [4] for the problem. At the same time, our algorithm is much simpler than the algorithms in $[4,23,25,33]$, illustrating the power of kernelization in the process of designing efficient algorithms for parameterized $\mathcal{N} \mathcal{P}$-hard problems. Finally, after a kind of race resulting in better and better algorithms [4, 23, 25, 33], Fomin and Thilikos recently presented an $O\left(n^{3}+2^{15.13 \sqrt{k}}\right)$ time algorithm to solve the PLANAR-DS problem based on the concept of branchwidth [25], the best treewidth based algorithm being only slightly worse [23]. 
7. Summary and extensions. In this paper we exhibited the first lower bound results on kernel sizes and, motivated by these findings, we strived to improve on the (still huge) constants involved in the known linear kernel for PLANAR-DS.

Are there other, possibly more sophisticated arguments for showing lower bounds on kernel sizes? In particular, it would be interesting to have arguments ruling out, for example, the existence of a kernel of size $o\left(k^{3}\right)$ in a situation when a kernel of size $\mathcal{O}\left(k^{3}\right)$ has been obtained. The algebra we used in the proof of Theorem 3.1 does not extend to such cases.

We mention that the concept of a black-and-white graph we used for deriving the kernel upper bound results for PLANAR-DS also allows us to exhibit a small kernel for a variation of the DOMINATING SET problem, called the RED/BLUE DOMINATING SET problem, as studied by Weihe [41]: Given a graph $G=(V, E)$, with $V$ partitioned into $V_{\text {red }} \cup V_{\text {blue }}$, and a positive integer $k$, is there a red/blue dominating set $D \subseteq V_{\text {red }}$ with $|D| \leq k$, i.e., $V_{\text {blue }} \subseteq N(D)$ ? Namely, if we consider the red vertices as "black" in our notation and the blue vertices as "white," and if we reanalyze our reduction rules, we can state the following corollary.

Corollary 7.1. Planar Red/Blue dominating SET admits a problem kernel of size $67 k$.

As exhibited in $[4,23]$, the possibly better known FACE COVER problem can be solved with the help of PLANAR RED/BLUE DOMINATING SET, by introducing "face vertices." However, we are still investigating if we could claim a small kernel for FACE COVER, since we are not keeping the (face) structure of the original problem. Notice that a cubic kernel was derived in [1, Thm. 1]. Based on this sort of problem kernel, we could then arrive at an $O^{*}\left(c^{\sqrt{k}}\right)$ algorithm for FACE COVER that is significantly better than what was obtained in [4], close to being competitive with [23], along the lines of the preceding section.

Appendix. In this section we prove Lemma 5.7. We first start with some observations and preliminary results.

Fix a region $R(v, w)$ between $v, w \in D$. Without loss of generality, assume the boundary of $R$ is determined by the two paths $\left(v, v_{1}, w_{1}, w\right)$ and $\left(v, v_{2}, w_{2}, w\right)$. Note that all vertices in $V(R)$ belong to $N(v, w)$, and that $v_{1}, v_{2} \in N^{*}(v)$ and $w_{1}, w_{2} \in$ $N^{*}(w)$. If there is a degree- 1 vertex $x$ connected to $v$ (resp., $w$ ), then this vertex is in $N_{3}(v)$ (resp., $N_{3}(w)$ ) and must be colored white by Rule 3. Similarly, if there exists a degree-2 vertex $y$ that is connected to $v$ and either $v_{1}$ or $v_{2}$ (resp., $w$ and either $w_{1}$ or $w_{2}$ ), then $y$ is in $N_{2}(v)$ (resp., $N_{2}(w)$ ) and must be colored white by Rule 3. Now if a degree- 1 white vertex is connected to $v$, then since the vertices in $N^{\dagger}(v)$ are white and are neighbors of $v$, by Rule 6 we must have $N^{\dagger}(v)=\emptyset$. During the process of counting the number of vertices in $N^{\dagger}(v)$, we bounded the number of vertices in $N^{\dagger}(v)$ by $3\left|N^{*}(v)\right| / 2$. This can be looked at as each vertex in $N^{*}(v)$ contributing $3 / 2$ vertices to $\left|N^{\dagger}(v)\right|$. So if a degree-1 white vertex is connected to $v$ (note that at most one degree- 1 vertex can be connected to $v$ ), this means that $N^{*}(v)$ which contains at least two vertices will not contribute to the number of vertices in $N^{\dagger}(v)$, and hence, the bound on $\left|N^{\dagger}(v)\right|$ will be decreased by at least three. Similarly, if a degree-2 white vertex is connected to $v$ and $v_{1}$ (or $v_{2}$ ) (again, note that there can be at most one degree- 2 vertex connected to $v$ and to $v_{1}$ (or $\left.v_{2}\right)$ ), then no vertex in $N^{\dagger}(v)$ can be connected to $v_{1}$ (or $v_{2}$ ). This can be regarded as a reduction to the bound on $\left|N^{\dagger}(v)\right|$ by $3 / 2$. Thus, if we use the upper bound on the number of vertices in category (2) computed above, we may assume without loss of generality that no degree- 1 vertex is connected to $v$ or $w$ and that no degree- 2 vertex is connected to $v$ and $v_{1}, v$ and $v_{2}$, 
$w$ and $w_{1}$, or $w$ and $w_{2}$. We will also assume that the boundary of a region $R(v, w)$ consists of exactly six distinct vertices; that is, the region is not a degenerated region. The case of a degenerated region obviously yields a better bound on the number of vertices in the region. Let us call a region with all the above properties nice. We start with the following propositions.

Proposition A.1. If there is no simple black vertex strictly inside a quasi-simple region $R=R(v, w)$, then $V(R)$ contains at most two simple white vertices.

Proof. Suppose, to get a contradiction, that $V(R)$ contains more than two simple white vertices, and let $a, b$, and $c$ be three such vertices. Since all three vertices are simple, one vertex must be engulfed within the area determined by $v, w$, and the other two vertices. Suppose that $b$ is situated within the area $(v, a, w, c, v)$. Since, by the assumption of the proposition, all the simple vertices strictly inside $V(R)$ must be white, and since all the nonsimple vertices inside $V(R)$ (i.e., vertices in $R^{+}$) are white by definition, and since no edges exist between white vertices, it follows that the white simple vertex $b$, engulfed by the area $(v, a, w, c, v)$, is connected only to $v$ and $w$ and hence has degree 2. Note that the color of both $v$ and $w$ must be black since there are simple white vertices that are connected to both $v$ and $w$. Now $|W(v, w)|>2$ because $\{a, b, c\} \subseteq W(v, w)$. But this makes Rule 8 applicable, contradicting the fact that $G$ is reduced. This completes the proof.

Proposition A.2. Let $R=R(v, w)$ be a quasi-simple region where the color of $v$ is black; then $V(R)$ has at most four simple vertices.

Proof. Suppose first that $R$ has six or more simple vertices. Let $S$ be the set of those simple vertices that are strictly inside $R$. Then $|S| \geq 4$. Since the vertices in $S$ are simple and hence connect to both $v$ and $w$, it is obvious that no vertex lying strictly inside $R$ can dominate all vertices in $S$. Since $S$ is a subset of those vertices in $N_{2}(v, w) \cup N_{3}(v, w)$ that are strictly inside $R$, it follows that no vertex that is strictly inside $R$ can dominate all vertices in $N_{2}(v, w) \cup N_{3}(v, w)$. Now all vertices that lie strictly inside $R$ belong to $N_{2}(v, w) \cup N_{3}(v, w)$; thus, by Rule 5 , all vertices strictly inside $R$ must be white. Noting that $|S| \geq 4$, and that all the vertices in $S$ are simple white vertices, this contradicts Proposition A.1.

Suppose now that $R$ has five simple vertices. Let $a, b$, and $c$ be the three simple vertices that lie strictly inside $R$. By an argument similar to the above, we can assume that vertex $b$ is engulfed within the area determined by $v, a, w$, and $c$. Again all the vertices strictly inside $R$ must belong to $N_{2}(v, w) \cup N_{3}(v, w)$. Since $a$ does not dominate $c$, and vice versa, it follows that $a$ and $c$ are colored white by Rule 5 . Now $a, b$, and $c$ are the only simple vertices strictly inside $R$, but by Proposition A.1, no three simple white vertices can be contained in $R$. This forces $b$ to be black and to be connected to both $a$ and $c$ (otherwise $b$ would be colored white by Rule 5). Now all other nonsimple vertices in $R$ must be connected to the boundary and hence cannot be connected to $b$ (all the vertices other than $a$ and $c$ which can be connected to $b$ have to belong to the area engulfed by $(v, a, w, c)$ and cannot be connected to the boundary). Thus, $W(b)=\{a, c\}$, and every vertex in $W(b)$ is connected to all vertices in $B(b)=\{v, w\}$ (note that since $a$ and $c$ are white and are connected to $w, w$ must be black). By Rule 7, $W(b)=\{a, c\}$ should have been removed at this point, a contradiction. Therefore, $R$ has at most four simple vertices and the proof is complete.

Proposition A.3. Let $(v, y, w, z, v)$ be the boundary of a quasi-simple region $R=R(v, w)$, and suppose that $v$ and $y$ are black. Then there can be at most one white vertex in $R^{+}=R^{+}(v, w)$ that is connected to both $v$ and $y$. 
Proof. Suppose, to get a contradiction, that there are at least two white vertices in $R^{+}$that are connected to both $v$ and $y$. Since all the vertices in $R^{+}$are white and hence cannot be connected together, there must exist two white vertices $a$ and $b$ in $R^{+}$satisfying that the area engulfed by $(v, a, y, v)$ is empty, and that the area engulfed by $(v, b, y, v)$ contains only the vertex $a$. Clearly, the degree of $a$ is exactly 2 , and $a$ belongs to $N_{2}(v) \cup N_{3}(v)$. Now since both $a$ and $b$ are connected to both $v$ and $y$, we have $N(a) \subseteq N(b)$. Given the fact that $v$ is black, this is a contradiction to Rule 6.

Proposition A.4. Let $R=R(v, w)$ be a quasi-simple region, and suppose that $v$ is black. Let $(v, y, w, z, v)$ be the boundary of $R$. If

(a) there are no simple vertices strictly inside $R$ or

(b) there are simple vertices strictly inside $R$ and all vertices in $R^{+}=R^{+}(v, w)$ are connected to $y$,

then $V(R) \cup\{w\}$ contains at most three white vertices. Moreover, if there are three white vertices in $V(R) \cup\{w\}$, then either $R^{+} \neq \emptyset$ or there is a simple black vertex interior to $R$.

Proof. To prove that part (a) implies the statement of the proposition, suppose that there are no simple vertices lying strictly inside $R$. Then clearly all the white vertices in $V(R)$ come from $R^{+} \cup\{y, z\}$. If $y$ is white, then no vertex in $R^{+}$can be connected to $y$ because the vertices in $R^{+}$are all white. On the other hand, since $R^{+} \subseteq N_{2}(v) \cup N_{3}(v)$, if $y$ is black, by Proposition A.3, at most one white vertex in $R^{+}$can be connected to $y$, and similarly if $z$ is black. Since every vertex in $R^{+}$has to be connected to either $y$ or $z$ by the definition of a quasi-simple region, it follows from the above that $V(R)$ contains at most two white vertices, and hence $V(R) \cup\{w\}$ contains at most three white vertices. Now when $V(R) \cup\{w\}$ contains three white vertices, $w$ must be white, and hence, $y$ and $z$ are black. Thus, the two white vertices other than $w$ in $V(R) \cup\{w\}$ come from $R^{+}$, and $R^{+} \neq \emptyset$.

To prove part (b), note first that, by Proposition A.2, the number of simple vertices in $R$ including $y$ and $z$ is bounded by four. We will assume that the number of simple vertices in $R$ is exactly four. The cases when there are less than four simple vertices in $R$ are simpler and yield the desired bound. Let $a$ and $b$ be the other two simple vertices, and assume that the four simple vertices $y, a, b, z$ appear in the preceding sequence in a clockwise order around $v$. Observe that the white vertices in $V(R)$ come from $R^{+} \cup\{y, a, b, z\}$. Also observe that since all the vertices in $R^{+}$ are connected to $y$ by the hypothesis of part (b), either $y$ is white and $R^{+}$is empty or $y$ is black and by Proposition A.3, $R^{+}$contains at most one vertex. It follows that the number of white vertices in $R^{+} \cup\{y\}$ is bounded by one. Now suppose to get a contradiction that $V(R) \cup\{w\}$ contains four white vertices. Since no two white vertices are connected and since all vertices in $\{a, b, z\}$ are connected to $w, w$ must be black, and all three vertices $a, b$, and $z$ must be white. But then the degree of $b$ is exactly 2 , and $|W(v, w)|>2$, contradicting Rule 8 . To complete the proof, supposing that $V(R) \cup\{w\}$ contains exactly three white vertices, we need to show that either $R^{+} \neq \emptyset$ or there exists a simple black vertex inside $R$. Suppose, to get a contradiction, that $R^{+}=\emptyset$ and the interior vertices to $R, a, b$, are all white. Then $w$ must be black in this case, and either $y$ or $z$ is white. Without loss of generality, assume $y$ is white. Since there are no edges between white vertices, the degree of $a$ must be 2 , and $\{y, a, b\} \subseteq W(v, w)$, again a contradiction to Rule 8 .

Lemma A.5 (see Lemma 5.7). Let $R=R(v, w)$ be a nice region in $V[\Re]$. The number of vertices in $V(R)$ is bounded by 16 . 
Proof. Every vertex in $V(R)$ is in $N(v, w)$, and hence is connected to either $v$ or $w$. We distinguish two cases.

Case 1. $N_{3}(v, w)=\emptyset$. In this case every vertex in $V(R)-\left\{v_{1}, v_{2}, w_{1}, w_{2}\right\}$ has to be connected to at least one vertex in $\left\{v_{1}, v_{2}, w_{1}, w_{2}\right\}$ because $v_{1}, v_{2}, w_{1}, w_{2}$ are the only vertices in $V(R)$ that possibly belong to $N_{1}(v, w)$. Since $R$ is nice, the vertices in $V(R)-\left\{v_{1}, v_{2}, w_{1}, w_{2}\right\}$ can be classified into the following categories, where a vertex is assigned to the first category that it satisfies:

(i) vertices connected to $v$ and $v_{1}$, but not connected to $w_{1}$;

(ii) vertices connected to $v$ and $w_{1}$;

(iii) vertices connected to $v$ and $w_{2}$;

(iv) vertices connected to $v$ and $v_{2}$;

(v) vertices connected to $w$ and $w_{2}$, but not connected to $v_{2}$;

(vi) vertices connected to $w$ and $v_{2}$;

(vii) vertices connected to $w$ and $v_{1}$; and

(viii) vertices connected to $w$ and $w_{1}$.

Note that one of categories (ii) and (vii) must be empty; otherwise, according to our placement of the vertices in the categories, we have two distinct vertices in $V(R)$ other than $v_{1}$ and $w_{1}$ : one of them is connected to $v$ and $w_{1}$ and the other to $w$ and $v_{1}$, contradicting the planarity of the graph. Similarly, one of categories (iii) and (vi) must be empty. Without loss of generality, assume that categories (iii) and (vii) are empty. If, in addition, either category (ii) or category (vi) is empty, then the situation becomes simpler, leading to a better bound on the number of vertices in $V(R)$. Thus, we will assume that both categories (ii) and (vi) are nonempty. Note also that since $R$ is nice, no vertex interior to $R$ has degree 2 , and every vertex in category (i) must be connected to some vertex interior to $R$. Since category (ii) is nonempty, and by the planarity of $G$, a vertex in category (i) can be connected only to vertices in category (ii). Moreover, since the vertices in category (i) are connected only to $v$ and to neighbors of $v$ including $v_{1}$ (since these vertices can be connected only to $v$ and to vertices in category (ii)), all these vertices belong to $N_{2}(v) \cup N_{3}(v)$. Since $v$ is black, by Rule 3 , all vertices in category (i) must be white. Now the vertices in categories (i) and (ii), plus $v, v_{1}$, and $w_{1}$, form a quasi-simple region between $v$ and $w_{1}, Q_{1}=R\left(v, w_{1}\right)$. Moreover, all the vertices in $V\left(Q_{1}\right)$, except those in category (i), are simple vertices because all vertices in $V\left(Q_{1}\right)$, except those in category (i), have to be connected to both $v$ and $w_{1}$. Since $v$ is black (all the endpoints of regions in $\Re$ are black), by Proposition A.2, the number of vertices in $V\left(Q_{1}\right)$ except those vertices in category (i) is bounded by 4 . Now we bound the number of vertices in category (i). Every vertex in category (i) is white and is connected to $v$ and $v_{1}$. If category (i) is nonempty, then $v_{1}$ must be black, and the vertices in category (i) are white vertices in $Q_{1}^{+}\left(v, w_{1}\right)$ that are connected to $v$ and $v_{1}$. It follows from Proposition A.3 that the number of vertices in category (i) is bounded by 1 . This shows that the number of vertices in $V\left(Q_{1}\right)$ is bounded by 5 . By symmetry, the number of vertices in $V\left(Q_{2}\right)$, where $Q_{2}$ is the quasi-simple region between $w$ and $v_{2}$ consisting of the vertices in categories (v) and (vi), plus the vertices $w, w_{2}, v_{2}$, is bounded by 5 . Now we bound the number of vertices in categories (iv) and (viii). We have the following claim.

Claim. The number of vertices in category (iv) (resp., category (viii)) is bounded by 2. Moreover, at most one vertex in category (iv) (resp., category (viii)) is white.

Consider the vertices in category (iv). Suppose that there are three or more vertices in category (iv), and let $a_{1}, a_{2}, a_{3}$ be three vertices in category (iv) such that no vertex is engulfed in the area of the embedding determined by $\left(v, a_{1}, v_{2}\right), a_{1}$ 


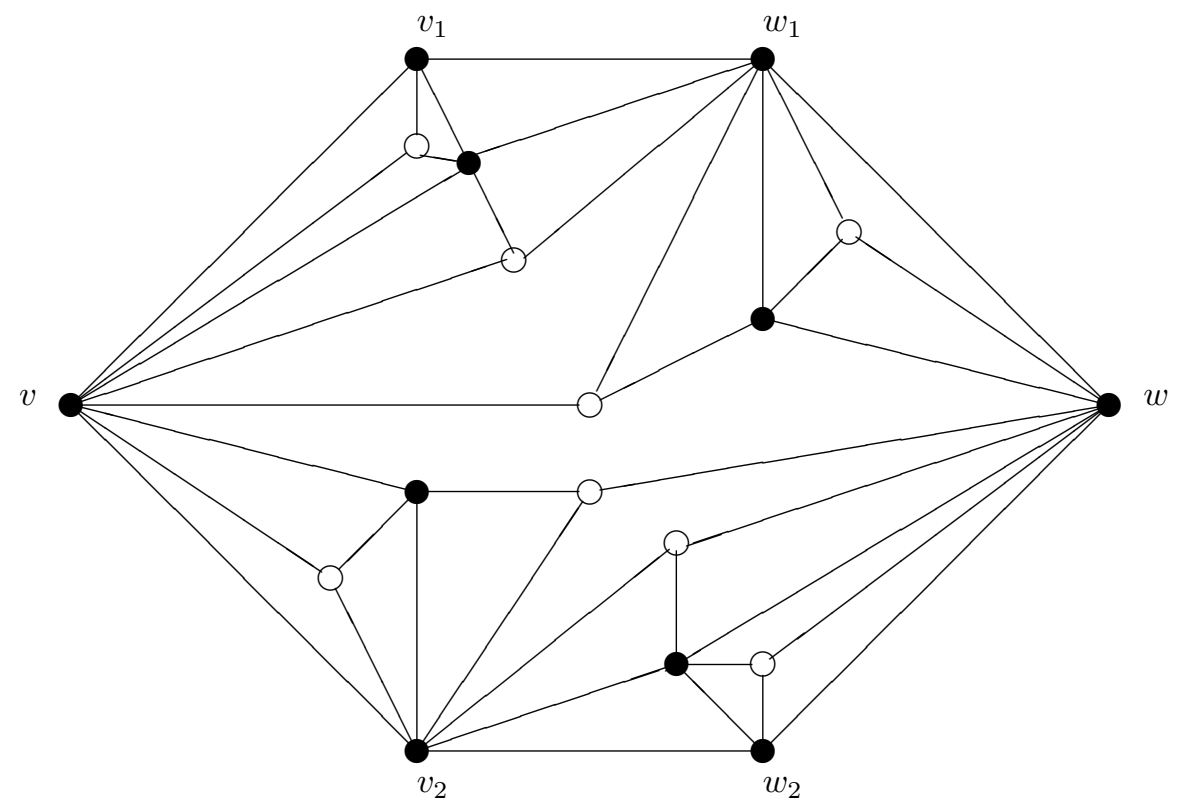

FIG. 4. Illustration of a possible worst-case scenario for Case 1. Empty circles represent white vertices, and filled circles black vertices.

is the only vertex engulfed in the area determined by $\left(v, a_{2}, v_{2}\right)$, and $a_{1}$ and $a_{2}$ are the only two vertices engulfed in the area determined by $\left(v, a_{3}, v_{2}\right)$. Now $a_{1}$ and $a_{2}$ must belong to $N_{2}(v) \cup N_{3}(v)$. Since $v$ is black, it follows from Rule 3 that $a_{1}$ and $a_{2}$ must be white and that no edge exists between $a_{1}$ and $a_{2}$. But this means that $N\left(a_{1}\right) \subseteq N\left(a_{2}\right)$, and since $a_{1} \in N_{2}(v)$ and $v$ is black, according to Rule 6 , this leads to a contradiction. It follows that at most two vertices can be in category (iv). By symmetry, at most two vertices can be in category (viii). Note also that it follows from the above proof that if there are exactly two vertices $a_{1}$ and $a_{2}$ in category (iv) (resp., category (viii)), then at most one vertex in $\left\{a_{1}, a_{2}\right\}$ can be white. This proves the claim.

Now the vertices in $V(R)$ consist of vertices of $V\left(Q_{1}\right)$, vertices of $V\left(Q_{2}\right)$, category (iv) and category (viii) vertices, and the two vertices $v_{2}$ and $w_{1}$ (in case these two vertices were not included in any of the other categories). It follows that the number of vertices in $R$ is bounded by 16 . See Figure 4 for an illustration of such a possible scenario.

Case 2. $N_{3}(v, w) \neq \emptyset$. Let $X$ be the set of white vertices in $N_{2}(v, w)$ that are in $V(R), Y$ the set of black vertices in $N_{2}(v, w) \cup N_{3}(v, w)$ that are in $V(R)$, and $Z$ the set of white vertices in $N_{3}(v, w)$ that are in $V(R)$. We first make a few observations.

Observation 1. $|X| \leq 7$. We first show that $|X| \leq 8$. Remove the vertices in $N_{3}(v, w)$ interior to $R$; then define categories (i)-(viii) as above. Similar to Case 1, we can assume that the vertices in categories (i) and (ii), plus the vertices $v, v_{1}$, and $w_{1}$, form a quasi-simple region $Q_{1}=R\left(v, w_{1}\right)$, and that those in categories (v) and (vi), plus the vertices $w, w_{2}$, and $v_{2}$, form a quasi-simple region $Q_{2}=R\left(w, v_{2}\right)$. Since $X \subseteq N_{2}(v, w)$, every vertex in $X$ must belong to one of categories (i)-(viii) or possibly to the set $\left\{v_{1}, v_{2}, w_{1}, w_{2}\right\}$. From the definition of categories (i) and (ii), vertices in category (i) form the set $Q_{1}^{+}=Q_{1}^{+}\left(v, w_{1}\right)$ in the quasi-simple region $Q_{1}$, and all the vertices in $Q_{1}^{+}$are connected to $v_{1}$. Now add the vertices in $N_{3}(v, w)$ back, and note 
that no black vertex in $N_{3}(v, w)$ that is not connected to $w_{1}$ resides in $Q_{1}$. The reason is that such a vertex would be in $N_{2}(v) \cup N_{3}(v)$ (otherwise, this vertex will have to be connected to $w_{1}$ - the only vertex in $Q_{1}$ possibly not in $N(v)$ ) and hence colored white by Rule 3. Now $Q_{1}$ plus the set of black vertices in $N_{3}(v, w)$ that reside in $Q_{1}$, minus the set of white vertices in $N_{3}(v, w)$ that reside in $Q_{1}$, satisfies condition (b) in Proposition A.4, and the number of white vertices in $Q_{1}$ is bounded by 3 . Since no two white vertices are connected together and hence the presence of the white vertices from $N_{3}(v, w)$ in $Q_{1}$ cannot increase the number of possible white vertices in $Q_{1}$, we conclude that the number of white vertices in $Q_{1}$ that are not in $N_{3}(v, w)$, and hence, the number of vertices in $X$ that belong to $Q_{1}$, is bounded by 3 . Similarly, the number of vertices in $X$ that belong to $Q_{2}$ is bounded by 3. Moreover, the statement of the claim in Case 1 carries in a straightforward manner to Case 2, and categories (iv) and (viii) contain at most one white vertex each. It follows that the number of white vertices in the set $X$ is bounded by 8 . Now if $|X|=8$, then both $Q_{1}$ and $Q_{2}$ (plus the black vertices in $N_{3}(v, w)$ that reside in $Q_{1}$ and $Q_{2}$ ) contain three white vertices. Since $Q_{1}$ contains exactly three white vertices, by Proposition A.4, either $Q_{1}^{+} \neq \emptyset$, or $Q_{1}$ must contain an interior black vertex. If $Q_{1}^{+} \neq \emptyset$, since $R$ is nice, the vertex in $Q_{1}^{+}$must be connected to some vertex interior to $Q_{1}$ which must be black because the vertices in $Q_{1}^{+}$are white. Therefore, if $Q_{1}$ contains exactly three white vertices, then there must exist an interior black vertex $p$ in $Q_{1}$. Similarly, there must exist an interior black vertex $q$ in $Q_{2}$. Since both $p$ and $q$ are black and are in $N_{2}(v, w) \cup N_{3}(v, w)$, by Rule $4, p$ and $q$ must dominate all vertices in $N_{3}(v, w) \neq \emptyset$. In particular, $p$ which is interior to $Q_{1}$ must dominate $q$ which is interior to $Q_{2}$. This is a contradiction to the planarity of the graph. It follows that $|X| \leq 7$.

Observation 2. Every vertex in $Y$ must dominate all vertices in $N_{3}(v, w)$.

This observation follows from Rule 4 since the vertices in $Y$ are black and are a subset of $N_{2}(v, w) \cup N_{3}(v, w)$.

Let $H$ be the graph obtained from $G$ by identifying the vertex $v$ with $w$ along the path $\left(v, v_{1}, w_{1}, w\right)$. Clearly, $H$ is planar. Let $u$ be the resulting vertex by this identification. Let $Y^{\prime}$ be the set of vertices in $Y$ that are in $H$, and let $y=\left|Y^{\prime}\right|$. Similarly, let $Z^{\prime}$ be the set of vertices in $Z$ that are in $H$, and let $z=\left|Z^{\prime}\right|$. Observe that the vertex $u$ is connected to all the vertices in $Y^{\prime}$ and $Z^{\prime}$ in $H$, and that the only vertices that have been removed by this identification are boundary vertices to $R$ that belong to $\left\{v_{1}, v_{2}, w_{1}, w_{2}\right\}$.

Observation 3. If $y>1$ and $z>1$, then the number of vertices in $V(R)$ is bounded by 16 .

Suppose that $y>1$ and $z>1$. If $y>2$, since every vertex in $Y^{\prime}$ must dominate the vertices in $Z^{\prime}$, it follows that the subgraph of $H$ induced by the set of vertices $Y^{\prime} \cup Z^{\prime} \cup\{u\}$ contains a copy of $K_{3,3}$, contradicting the planarity of $H$ (the vertices in $Y^{\prime}$ form the first bipartition, and the other vertices form the second bipartition). Suppose now that $y=2$. If $z>2$, then similarly, the subgraph induced by $Z^{\prime} \cup\{u\} \cup Y^{\prime}$ contains a copy of $K_{3,3}$ (the vertices in $Y^{\prime} \cup\{u\}$ form the first bipartition, and those in $Z^{\prime}$ form the second bipartition). Suppose now that $y=z=2$. Then the number of vertices in $X \cup Y^{\prime} \cup Z^{\prime}$ is bounded by 11. Since $|V(R)| \leq\left|X \cup Y^{\prime} \cup Z^{\prime} \cup\left\{v_{1}, v_{2}, w_{1}, w_{2}\right\}\right|$, it follows that the number of vertices in $V(R)$ is bounded by 16 .

Now we distinguish the following two subcases.

Subcase 2.1. $z \leq 1$. Let $Y_{1}=Y^{\prime} \cap N_{2}(v, w)$ be the set of black vertices in $Y^{\prime}$ that are in $N_{2}(v, w), y_{1}=\left|Y_{1}\right|, Y_{2}=Y^{\prime}-Y_{1}$ be the set of black vertices in $Y^{\prime}$ that are in $N_{3}(v, w)$, and $y_{2}=\left|Y_{2}\right|$. Note that every vertex in $Y^{\prime}$ must be connected to all vertices 
in $Y_{2} \cup Z$ by Rule 4. If $y=y_{1}+y_{2}<5$, then since $z \leq 1$ and the number of vertices in $X$ is bounded by 7 by Observation 1 , the number of vertices in $V(R)$ is bounded by 16 . So we can assume that $y \geq 5$. If $y_{2}+z \geq 4$, then the subgraph induced by the vertices $\{u\} \cup Y_{2} \cup Z$ is a copy of $K_{5}$. Thus, $y_{2}+z<4$. If $y_{2}+z=3$, then the subgraph induced by the bipartition $\left(Y_{2} \cup Z, Y_{1} \cup\{u\}\right)$ contains a copy of $K_{3,3}$, whereas if $y_{2}+z=2$, then the subgraph induced by the bipartition $\left(\{u\} \cup Y_{2} \cup Z, Y_{1}\right)$ contains a copy of $K_{3,3}$. Suppose now that $y_{2}+z=1$. If $y_{1} \leq 4$, then $y+z \leq 5$, and hence, the number of vertices in $R$ is bounded by 16 . Assume now that $y_{1} \geq 5$. Let $p$ be the vertex in $Y_{2} \cup Z$; then $p$ is connected to all vertices in $Y_{1}$ in $H$ and hence in $G$. Moreover, every vertex in $Y_{1}$ is connected to either $v$ or $w$ (or both) in $G$. Since $y_{1} \geq 5$, there must exist at least three vertices in $Y_{1}$ that are connected to either $v$ or to $w$ in $G$. Let these vertices be $p_{1}, p_{2}$, and $p_{3}$, and assume, without loss of generality, that these vertices are connected to $v$. Since $p_{1}, p_{2}$, and $p_{3}$, are also connected to $p$, there must exist a vertex in $\left\{p_{1}, p_{2}, p_{3}\right\}$, say $p_{2}$, that is interior to the region determined by $v, p$, and the other two vertices. But $p_{2} \in Y_{1} \subseteq N_{2}(v, w)$, and hence $p_{2}$ must be connected to the boundary of $R$ (because $p_{2}$ must be connected to some vertex in $N_{1}(v, w)$ ), a contradiction. Thus, the number of vertices in $V(R)$ is bounded by 16 .

Subcase 2.2. $y \leq 1$. If $z \leq 4$, then $y+z \leq 5$, and given that $|X| \leq 7$ by Observation 1, the total number of vertices in $V(R)$ is bounded by 16. Suppose now that $z \geq 5$. Observe first that $Y \neq \emptyset$; otherwise, $N_{2}(v, w) \cup N_{3}(v, w)$ consists of only white vertices, and $N_{3}(v, w)$, which contains at least five white vertices ( $Z \subseteq$ $N_{3}(v, w)$ ), could not be dominated by a single vertex in $N_{2}(v, w) \cup N_{3}(v, w)$. This would make Rule 2 applicable, a contradiction. Let $p_{1}, p_{2}, p_{3}, p_{4}$, and $p_{5}$ be vertices in $Z$. Since each of these vertices must be connected to either $w$ or $v$, at least three vertices in $\left\{p_{1}, p_{2}, p_{3}, p_{4}, p_{5}\right\}$ are connected to either $v$ or $w$. Suppose, without loss of generality, that $\left\{p_{1}, p_{2}, p_{3}\right\}$ are connected to $v$, and note that by Observation 2, every vertex in $Y$ must be connected to all vertices in $Z$. If $|Y| \geq 2$, then $(\{v\} \cup$ $\left.Y,\left\{p_{1}, p_{2}, p_{3}\right\}\right)$ would be a copy of $K_{3,3}$. Suppose now that $|Y|=1$, and let $q$ be the single vertex in $Y$. Since $p_{1}, p_{2}, p_{3}$ are white and belong to $N_{3}(v, w)$, these vertices can connect only to vertices in $\{v, q, w\}$. Again, by planarity, at least one vertex in $\left\{p_{1}, p_{2}, p_{3}\right\}$ is not connected to $w$ and hence must be of degree 2 . But then $|W(v, q)| \geq 3$, and $W(v, q)$ contains a degree- 2 vertex. This contradicts Rule 8 .

It follows that in all cases the number of vertices in $V(R)$ is bounded by 16 . This completes the proof.

\section{REFERENCES}

[1] F. Abu-Khzam and M. Langston, A direct algorithm for the parameterized face cover problem, in Proceedings of the 1st International Workshop on Parameterized and Exact Computation, Lecture Notes in Comput. Sci. 3162, Springer, Berlin, 2004, pp. 213-222.

[2] F. Abu-Khzam, M. Langston, and P. Shanbhag, A High-Performance Toolkit for Fast Exact Algorithms, invited talk to the Workshop on Fixed-Parameter Tractability, Ottawa, ON, Canada, 2003; available online at http://citeseer.ist.psu.edu/560785.html.

[3] J. Alber, N. Betzler, And R. Niedermeier, Experiments on data reduction for optimal domination in networks, Ann. Oper. Res., 146 (2006), pp. 105-117.

[4] J. Alber, H. Bodlaender, H. Fernau, T. Kloks, and R. Niedermeier, Fixed parameter algorithms for dominating set and related problems on planar graphs, Algorithmica, 33 (2002), pp. 461-493.

[5] J. Alber, M. Fellows, And R. Niedermeier, Polynomial-time data reduction for dominating set, J. ACM, 51 (2004), pp. 363-384.

[6] J. Alber, H. Fernau, and R. Niedermeier, Parameterized complexity: Exponential speedup for planar graph problems, J. Algorithms, 52 (2004), pp. 26-56. 
[7] J. Alber, H. Fernau, and R. Niedermeier, Graph separators: A parameterized view, J. Comput. System Sci., 67 (2003), pp. 808-832.

[8] B. BAKER, Approximation algorithms for NP-complete problems on planar graphs, J. ACM, 41 (1994), pp. 153-180.

[9] R. Balasubramanian, M. R. Fellows, and V. Raman, An improved fixed parameter algorithm for vertex cover, Inform. Process. Lett., 65 (1998), pp. 163-168.

[10] J. Cheetham, F. Dehne, A. Rau-Chaplin, U. Stege, and P. J. Taillon, Solving large FPT problems on coarse grained parallel machines, J. Comput. System Sci., 67 (2003), pp. 691-706.

[11] J. Chen And I. Kanj, Constrained minimum vertex cover in bipartite graphs: Complexity and parameterized algorithms, J. Comput. System Sci., 67 (2003), pp. 833-847.

[12] J. Chen and I. KanJ, Improved exact algorithms for MaX-Sat, Discrete Appl. Math., 142 (2004), pp. 17-27.

[13] J. Chen, I. Kanj, And W. JiA, Vertex cover: Further observations and further improvement, J. Algorithms, 41 (2001), pp. 280-301.

[14] J. Chen, I. KANJ, AND G. XIA, Improved parameterized upper bounds for vertex cover, in Proceedings of the 31st International Symposium on the Mathematical Foundations of Computer Science, Lecture Notes in Comput. Sci. 4162, Springer, Berlin, 2006, pp. 238-249.

[15] F. Dehne, M. Fellows, H. Fernau, E. Prieto, and F. Rosamond, Nonblocker: Parameterized algorithmics for Minimum Dominating Set, in Software Seminar SOFSEM, Lecture Notes in Comput. Sci. 3831, Springer, Berlin, 2006, pp. 237-245.

[16] R. Diestel, Graph Theory, Springer, Berlin, 1997.

[17] R. Downey and M. Fellows, Parameterized Complexity, Springer, New York, 1999.

[18] R. Downey, M. Fellows, and U. Stege, Parameterized complexity: A framework for systematically confronting computational intractability, in Contemporary Trends in Discrete Mathematics, DIMACS Ser. Discrete Math. Theoret. Comput. Sci. 49, R. Graham, J. Kratochvíl, J. Nešetřil, and F. Roberts, eds., AMS, Providence, RI, 1999, pp. 49-99.

[19] M. Fellows, Parameterized complexity: The main ideas and connections to practical computing, in Electronic Notes in Theoretical Computer Science, Vol. 61, 2002.

[20] M. Fellows, C. McCartin, F. Rosamond, and U. Stege, Coordinatized kernels and catalytic reductions: An improved FPT algorithm for max leaf spanning tree and other problems, in FST TCS 2000, Lecture Notes in Comput. Sci. 1974, Springer, Berlin, 2000, pp. 240-251.

[21] H. Fernau, On parameterized enumeration, in Computing and Combinatorics, Lecture Notes in Comput. Sci. 2387, Springer, Berlin, 2002, pp. 564-573.

[22] H. Fernau, ROMAN Domination: A parameterized perspective, in Software Seminar SOFSEM 2006, Lecture Notes in Comput. Sci. 3831, Springer, Berlin, 2006, pp. 262-271.

[23] H. Fernau And D. Juedes, A geometric approach to parameterized algorithms for domination problems on planar graphs, in Mathematical Foundations of Computer Science 2004, Lecture Notes in Comput. Sci. 3153, Springer, Berlin, 2004, pp. 488-499.

[24] H. Fernau And R. Niedermeier, An efficient exact algorithm for constraint bipartite vertex cover, J. Algorithms, 38 (2001), pp. 374-410.

[25] F. V. Fomin AND D. M. Thilikos, Dominating sets in planar graphs: Branch-width and exponential speed-up, in Proceedings of the Fourteenth ACM-SIAM Symposium on Discrete Algorithms (SODA), SIAM, Philadelphia, 2003, pp. 168-177.

[26] F. V. Fomin AND D. ThiLikos, Fast parameterized algorithms for graphs on surfaces: Linear kernel and exponential speed-up, in Automata, Languages and Programming (ICALP), Lecture Notes in Comput. Sci. 3142, Springer, Berlin, 2004, pp. 581-592.

[27] M. Garey and D. Johnson, Computers and Intractability: A Guide to the Theory of NPCompleteness, Freeman, San Francisco, 1979.

[28] J. Gimbel and C. Thomassen, Coloring triangle-free graphs with fixed size, Discrete Math., 219 (2000), pp. 275-277.

[29] H. Grötzsch, Ein Dreifarbensatz für dreikreisfreie Netze auf der Kugel, Wiss. Z. MartinLuther-Univ. Halle-Wittenberg Ma.-Nat. Reihe, 8 (1958/1959), pp. 109-120.

[30] F. Henglein and H. G. Mairson, The complexity of type inference for higher order typed lambda calculi, J. Funct. Programming, 4 (1994), pp. 435-477.

[31] J. Hopcroft and R. TARJAn, Efficient planarity testing, J. ACM, 21 (1974), pp. 549-568.

[32] R. Hyafil and R. L. Rivest, Constructing optimal binary trees is NP-complete, Inform. Process. Lett., 5 (1976), pp. 15-17.

[33] I. Kanj And L. Perković, Improved parameterized algorithms for planar dominating set, in Mathematical Foundations of Computer Science 2002, Lecture Notes in Comput. Sci. 2420, Springer, Berlin, 2002, pp. 399-410.

Copyright (c) by SIAM. Unauthorized reproduction of this article is prohibited. 
[34] S. Кнот And V. RAman, Parameterized complexity of finding subgraphs with hereditary properties, Theoret. Comput. Sci., 289 (2002), pp. 997-1008.

[35] O. Lichtenstein And A. Pneuli, Checking that finite-state concurrent programs satisfy their linear specification, in Proceedings of the 12th ACM SIGACT-SIGPLAN Symposium on Principles of Programming Languages (POPL), 1985, pp. 97-107.

[36] R. Niedermeier and P. Rossmanith, A general method to speed up fixed-parameter-tractable algorithms, Inform. Process. Lett., 73 (2000), pp. 125-129.

[37] O. Ore, Theory of Graphs, Amer. Math. Soc. Colloq. Publ. XXXVIII, AMS, Providence, RI, 1962.

[38] E. Prieto AND C. Sloper, Either/or: Using vertex cover structure in designing FPTalgorithms - the case of k-Internal Spanning Tree, in Algorithms and Data Structures (WADS), Lecture Notes in Comput. Sci. 2748, Springer, Berlin, 2003, pp. 474-483.

[39] C. Thomassen, Grötzsch's 3-color theorem and its counterparts for the torus and the projective plane, J. Combin. Theory Ser. B, 62 (1994), pp. 268-279.

[40] R. Uehara, Probabilistic Algorithms and Complexity Classes, Ph.D. thesis, Department of Computer Science and Information Mathematics, The University of ElectroCommunications, Japan, 1998.

[41] K. WeIne, Covering trains by stations or the power of data reduction, in Proceedings of the Workshop on Algorithms and Experiments (ALEX '98), 1998, pp. 1-8; available online at http://rtm.science.unitn.it/alex98/proceedings.html.

Copyright ( by SIAM. Unauthorized reproduction of this article is prohibited. 